\title{
IMPLICATIONS OF CHIRAL DYNAMICS IN THEORIES
}

OF TECHNICOLOUR

I. ELEMENTARY COUPLINGS

$$
\begin{gathered}
\text { S. Chadha } \\
\text { CERN--Geneva } \\
\text { M.E. Peskin } \left.{ }^{*}\right) \\
\text { Cornell University } \\
\text { Ithaca, New York }
\end{gathered}
$$

ABSTRACT

\begin{abstract}
Models of dynamical weak interaction symmetry breaking involve new strong interactions with a mass scale of order $1 \mathrm{TeV}$. However, such models usually include multiplets of mesons - the Goldstone bosons accompanying the dynamical chiral symmetry breakdown - which have masses small compared to this scale and possibly as small as 10 $\mathrm{GeV}$. We construct an effective Lagrangian which describes the couplings of these mesons to one another and to the gauge bosons of colour and the electroweak interactions. As an application of this formalism, we compute the decay width of the $Z^{0}$ into pairs and triplets of these mesons.
\end{abstract}

*) Research supported by the National Science Foundation under grant PHY-77-22336.

Ref.Th.3023-CERN

29 January 1981 


\section{INTRODUCTION}

In the original gauge theories of the electroweak interactions ${ }^{1)}$, the intermediate vector bosons obtained their masses by a spontaneous breakdown of the gauge symmetry caused by the action of weakly interacting elementary scalar fields. For a variety of reasons, however, attention has recently been drawn to the possibility that this spontaneous symmetry breakdown may be a consequence of strong interaction dynamics. In this case, the electroweak theory need contain no elementary scalar fields; the Goldstone bosons associated with the spontaneous breakdown of the electroweak gauge symmetry are formed as bound states of the strongly interacting particles. It is by now generally recognized that the successful implementation of this idea to produce the correct masses of the weak gauge bosons requires a new set of gauge interactions which we will call technicolour, with a mass scale $M_{T C}$ of order $1 \mathrm{TeV}$ 2),3). Just as the $u$ and $d$ quarks, which participate in the usual strong interactions have a chiral SU(2) $\times$ $\mathrm{SU}(2)$ symmetry which is dynamically broken to the $\mathrm{SU}(2)$ of isospin invariance, one can arrange that the fermions which interact through technicolour have the electroweak symmetries $\mathrm{SU}(2) \times \mathrm{U}(1)$ as chiral symmetries, and that these symmetries are broken dynamically to the $U(1)$ invariance of electromagnetism. .

Realistic models of technicolour, however, entail sizeable multiplets of fermions interacting with technicolour. The group of chiral symmetries of these fermions is not simply $S U(2) \times U(1)$, but a much larger group which we will call G. The spontaneous breakdown of $G$ through strong interaction dynamics yields numerous Goldstone bosons. Although phenomenology requires that none of these. bosons remains exactly massless, all these bosons still remain light compared with $M_{T C}$. These particles are the most obvious and characteristic manifestation of the new technicolour strong interactions. It is therefore worth developing a formalism which can predict the properties of these particles - not only their masses, but also their interactions. The purpose of this paper is to provide a formalism for computing the couplings of these bosons to the electroweak gauge bosons, to colour gluons and to one another. We will not discuss the coupling of these bosons to ordinary quarks and leptons, since the understanding of the more elementary interaction between technicoloured and ordinary fermions 4 , 5) is still in a rather unsatisfactory state.

Our methodology will be a generalization of one which has proved useful in the ordinary strong interactions for describing the interactions of pions and kaons - the method of effective Lagrangians ${ }^{6)}$. This method is phenomenological: one considers that there is a separate field associated with each particle of mass much smaller than the natural strong interaction mass scale (in our case, $\left.\mathrm{M}_{\mathrm{TC}}\right)$. One allows these fields to have arbitrary interactions, subject to the 
constraints imposed by the chiral invariance $G$. However, by realizing the symmetry $G$ non-linearly on these fields, the constraint of $G$ invariance may be made a very stringent one. We will make use of a particularly elegant scheme for realizing $G$, devised by Callan, Coleman, Wess and Zumino ${ }^{7}$, which makes the analysis of these constraints straightforward. In the class of models we will study in this paper, the interactions among technicolour Goldstone bosons which are important at energies much less than $\mathrm{M}_{\mathrm{TC}}$ are completely determined in terms of only two parameters of the strong interaction theory, one of which may be determined from the Fermi constant $G_{F}$ or the $W$ boson mass. For readers familiar with the use of effective Lagrangians in pion physics, we should remark that we use the effective Lagrangian not as an adjunct to the use of current algebra, but rather as a complete and self-contained description of low-energy couplings. In taking this view, we follow a philosophy recently emphasized by Schwinger ${ }^{8)}$ and Weinberg ${ }^{9)}$. The utility of effective Lagrangian methods in theories of technicolour has been emphasized by Weinberg ${ }^{2)}$ and, more recently, by Beg, Politzer and Ramond ${ }^{10)}$.

To introduce our analysis, let us clarify the nature of the particles which appear in the effective Lagrangian. If we could view the technicolour forces isolated from all other interactions, we would simply consider the chiral symmetry $G$ to be spontaneously broken to a subgroup $\mathrm{H}$, producing a massless Goldstone boson for each generator of $\mathrm{G} / \mathrm{H}$. However, in order for a technicolour theory to describe the electroweak symmetry breakdown, fermions coupled to technicolour must also be coupled to the electroweak interactions. They may also be coupled to the colour gauge bosons of the ordinary strong interactions. These two additional couplings (of strength $e$ and $g_{S}$ respectively) are weak at the $1 \mathrm{TeV}$ energy scale of technicolour, but they nevertheless have an important effect on the spectrum of light mesons. The group of these weakly gauged symmetries $\mathrm{G}_{\mathrm{W}}=\mathrm{SU}(2) \times \mathrm{U}(1) \times \mathrm{SU}(3)$ will, in general, be a subgroup of $G$. Because this gauging singles out specific generators of $G$, it explicitly breaks $G$ to a smaller group $S$, the product of $G_{W}$ and those additional elements of $G$ which commute with all elements of $G_{W}$.

Weinberg ${ }^{2)}$ has argued that the perturbation caused by gauging $G_{W}$, divides the Goldstone bosons into three classes and the $G_{W}$ gauge bosons into two classes, depending on the relation of the corresponding generators of $G$ to the subgroup $H$, which is not spontaneously broken, and to the subgroup $S$ which is not explicitly broken. The gauge bosons corresponding to generators of $\mathrm{H}$ as well as $G_{W}$ remain massless; the other gauge bosons correspond to spontaneously broken generators of $G_{W}$ and acquire masses of order $e M_{T C}$ or $g_{S} M_{T C}$ through the Higgs mechanism. For the Goldstone bosons, the division is slightly more complex: 
a) To those generators of $G / H$ which are also generators of $G_{W}$, correspond fictitious Goldstone bosons, bosons which combine with vector mesons to give them mass through the Higgs mechanism.

b) To those generators of $G / H$ which are generators of $S$, but not $G_{W}$, correspond true Goldstone bosons, precisely massless spin-zero mesons.

c) To those generators of $G / H$ which are explicitly broken by the $G_{w}$ couplings, correspond pseudo-Goldstone bosons; these can acquire masses of order $\mathrm{eM}_{\mathrm{TC}}$ or $\mathrm{g}_{\mathrm{s}} \mathrm{M}_{\mathrm{TC}}$. (We will often abbreviate "technicolour pseudo-Goldstone bosons" to "technions".)

Note that even the heaviest of these particles have masses which are parametrically small compared to $\mathrm{M}_{\mathrm{TC}}$; hence all of these particles should appear in the effective Lagrangian. Indeed, we will see that this Lagrangian rather explicitly partitions them into their natural classes.

Our analysis will be set out as follows. In Section 2 we will describe the class of technicolour models which we will subject to analysis and present two specific models which will be used as illustrations in the course of our exposition. In Section 3 we will begin our study of effective Lagrangians by introducing the methodology and constructing an effective Lagrangian describing the unperturbed technicolour interactions. In Section 4 we will introduce the couplings of the gauge mesons of $\mathrm{G}_{\mathrm{w}}$ to technicolour Goldstone bosons. In Section 5 we will discuss the effect of the $G_{W}$ couplings on the Goldstone boson self-interactions, recovering, in the process, the formula for the technion masses. Finally, in Section 6 we will work out some phenomenological consequences of our effective Lagrangian, in particular its predictions for technion production at the $\mathrm{Z}^{0}$. We should note that our method does omit one class of couplings: since we do not work out the quantum modifications of chiral symmetries, we will not find the couplings of one Goldstone boson to two gauge particles which follow from the axial vector anomalies of $G$ currents. These interactions contribute to processes (such as $\pi^{0} \rightarrow 2 \gamma$ in the usual strong interactions) for which the effective Lagrangian vertices vanish. Some of these processes are interesting for phenomenology. These anomalous couplings are listed in Ref. 13); indeed, their phenomenological role has already been discussed in some detail 11),12),13).

The result of our analysis will be a catalogue (barring the exception just noted) of the couplings of the Goldstone bosons which result from the technicolour chiral symmetry breakdown. These couplings may then be used to study higher order corrections to technicolour interactions. To illustrate the role of these couplings, an illustrative example of such a correction will be worked out in our second paper ${ }^{14)}$. 
2. SOME FEATURES OF TECHNICOLOUR MODELS

In this Section we will review some general properties of technicolour theories and some features of a specific set of illustrative models. This review is intended to make our two papers self-contained; it will, however, be rather cursory. The reader who would like further details should consult Refs. 11), 15) and 16) and the general reviews of technicolour ${ }^{17 /, 18)}$. Our notation will conform to that of Ref. 15).

In constructing a theory in which technicolour interactions break the weakinteraction $\mathrm{SU}(2) \times \mathrm{U}(1)$ symmetry, one must take care that the pattern of symmetry breaking preserves the phenomenologically correct relations $m_{W}=m_{Z} \cos \theta_{W}$, $m_{\gamma}=0$. Weinberg and Susskind ${ }^{3)}$ showed that this may be accomplished by the following simple prescription: one sets the technicolour interactions of technifermions to be vectorlike and then one assigns to pairs of technifermions the $S U(2) \times U(1)$ quantum numbers of a quark or lepton doublet. Weinberg and Susskind argued that, in this case, the chiral symmetry breaking accompanying the technicolour strong interactions naturally induces the correct pattern of $S U(2) \times U(1)$ breaking. Sikivie, Susskind, Voloshin and Zakharov ${ }^{19)}$ have found more general scenarios which give the correct pattern of weak boson masses, but we will concentrate on the consequences of this simplest one.

The fact that the technicolour coupling in the Weinberg-Susskind scenario is vectorlike, leads us to expect particularly simple patterns of chiral symmetry breaking ${ }^{11), 15)}$. We will find it useful to build into our analysis some particular aspects of this simplification. Let us first recall these patterns. If the technifermions transform according to a complex representation of the technicolour group, one has, for $N$ multiplets of technifermions, a chiral symmetry $G=$ $=\mathrm{SU}(\mathrm{N}) \times \mathrm{SU}(\mathrm{N}) \times \mathrm{U}(1)$ which should be spontaneously broken to $\mathrm{H}=\mathrm{SU}(\mathrm{N}) \times \mathrm{U}(I)$. However, if the technifermions transform according to a real representation of the technicolour group, left-handed technifermions and the antiparticles of righthanded technifermions are equivalent objects which may be mixed by unitary transformations. This enlarges the chiral group to $G=S U(2 N)$. Dynamical generation of masses for technifermions breaks this group to $0(2 \mathrm{~N})$ or $S p(2 N)$, depending upon whether the representation to which the technifermions belong has a symmetric or antisymmetric quadratic invariant.

In all of these cases, the quotient space $G / H$ is a symmetric space. This implies that the symmetry breaking respects a parity invariance. Let $\left\{T_{i}\right\}$ be the generators of the unbroken subgroup $H$ and $\left\{X_{z}\right\}$ be the remaining, broken generators of $G$. Then there is a matrix $P$ such that 


$$
P T_{i} P=+T_{i}, \quad P X_{z} P=-X_{z} .
$$

It also implies that the $\mathrm{x}_{\mathrm{z}}$ comprise a single irreducible representation of H. These dual restrictions will be incorporated into our formalism from the beginning and will provide us with considerable simplifications.

Aside from these restrictions, our formalism will apply quite generally to any theory of technicolour Goldstone bosons. However, it will be useful at some points to provide concrete illustrations of our formalism within specific models. For this purpose, we will refer to two models - built actually out of the same scheme of couplings - whose properties we will now recall. These models were introduced by Dimopoulos in Ref. 11).

An interesting realization of the scheme of Weinberg and Susskind is found by assigning to technifermions vectorlike couplings to technicolour and the couplings to strong, weak and electromagnetic interactions which characterize a generation of quarks and leptons. We then have four weak doublets

$$
\left(\begin{array}{l}
N \\
E
\end{array}\right),\left(\begin{array}{l}
U_{a} \\
D_{a}
\end{array}\right) \quad a=1,2,3,
$$

where the index a labels the colour under the SU(3) of the ordinary strong interactions. Since there are, in all, eight species of technifermions, the global chiral symmetry of the technicolour interactions is either $G=\operatorname{SU}(8) \times$ $\times \mathrm{SU}(8) \times U(1)$ or $\mathrm{G}=\mathrm{SU}(16)$, depending upon whether the technifermions belong to a complex or a real representation of technicolour. In the first case, $H=$ $=\mathrm{SU}(8) \times \mathrm{U}(1)$; in the second, $\mathrm{H}=0(16)$ or $\mathrm{Sp}(16)$. The case of breaking to $\mathrm{Sp}(16)$ has been shown to have phenomenological difficulties ${ }^{15), 16)}$; let us simply discard it. The remaining two cases provide our two illustrative models which we will refer to henceforth as the SU and 0 models respectively.

To specify these models more precisely, let us write out explicitly the coupling of the technifermions (2.2) to $G_{W}=S U(3) \times S U(2) \times U(1)$. Since the generators of $G_{W}$, in the case of the 0 model, lie in the $S U(8) \times S U(8) \times$ $x U(1)$ subgroup of $S U(16)$, this coupling is identical in the two cases. It is useful to consider the charge conjugates of right-handed fermions as the more fundamental and so to represent the $G_{W}$ couplings using $16 \times 16$ matrices acting on the 16 component left-handed fermion field

$$
\psi_{0}=\left(U_{L}, D_{L}, N_{L}, E_{L},\left(U_{R}\right)^{c},\left(D_{R}\right)^{c},\left(N_{R}\right)^{c},\left(E_{R}\right)^{c}\right)(2.3)
$$


These matrices divide naturally into $8 \times 8$ blocks; purely left-handed and right-handed SU(8) transformations are generated by matrices of the form

$$
\left(\begin{array}{l|l}
H & 0 \\
\hline 0 & 0
\end{array}\right) \quad \text { and } \quad\left(\begin{array}{c|c}
0 & 0 \\
\hline 0 & -H^{T}
\end{array}\right) \text {, }
$$

respectively, with $\mathrm{H}$ Hermitian and traceless. The sets of eight components divide again into sets of six and two, corresponding to coloured and coloursinglet technifermions. Using this notation, the coupling of $\psi_{0}$ to $G_{w}$ is accomplished by using in the Lagrangian for $\psi_{0}$ the covariant derivative incorporating the $\mathrm{SU}(3), \mathrm{SU}(2)$ and $\mathrm{U}(1)$ gauge fields $A_{\mu}^{\alpha}, W_{\mu}^{a}, B_{\mu}$ :

$$
\begin{aligned}
& \underline{D}_{\mu} \psi_{0}=\partial_{\mu} \psi_{0}-i g_{s} A_{\mu}^{\alpha}\left(\begin{array}{c|c|c}
1_{2} \otimes \lambda_{\alpha} & 0 & 0 \\
\hline 0 & 0 & 0 \\
\hline 0 & \frac{-1_{2} \otimes \lambda_{\alpha}^{T}}{0} \frac{10}{0}
\end{array}\right) \\
& -i g W_{\mu}^{a}\left(\begin{array}{c|c}
\tau_{a} \otimes 1_{4} & 0 \\
\hline 0 & 0
\end{array}\right)
\end{aligned}
$$

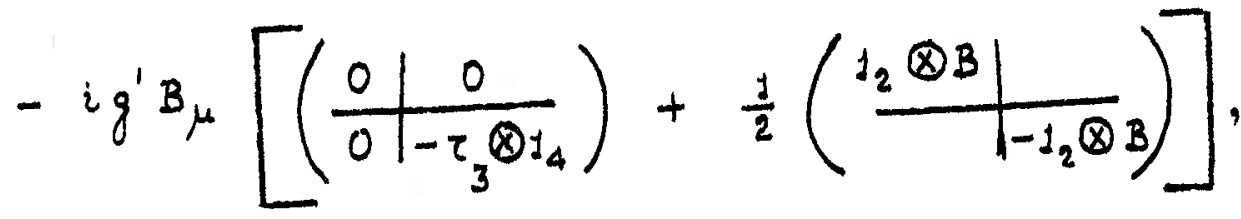

where $\tau_{a}$ and $\lambda_{\alpha}$ are generators of $S U(2)$ and $S U(3)$ respectively, $I_{n}$ is the $\mathrm{n} \times \mathrm{n}$ unit matrix and $\mathrm{B}$ is the $4 \times 4$ matrix

$$
B=\left(\begin{array}{ll|l}
1 / 3 & 1 / 3 & \\
\hline & -1
\end{array}\right)
$$

Our group theoretic conventions are summarized in the Appendix; the reader may find it useful to consult this Appendix as we pursue our analysis.

In the SU model, the breaking of $S U(8) \times S U(8) \times U(1)$ to $S U(8) \times U(1)$ yields 63 Goldstone bosons, all of definite odd-parity with respect to the technicolour couplings. For the most part, the quantum numbers of these particles may be worked out by pairing technifermions with anti-technifermions, though one must 
take care at the end to form combinations which are orthogonal to the axial vector $U(I)$, excluded from $G$ by its technicolour anomaly. One may check that, of the 63 bosons, there are seven colour singlet combinations. The remaining states are colour triplets (e.g., $\overline{\mathrm{N}}_{\mathrm{a}}$ ) and colour octets (e.g., $\overline{\mathrm{U}}_{\mathrm{a}} \mathrm{D}_{\mathrm{b}}$ ). The masses of these states are dominated by the contribution from one gluon exchange

$$
\left.m^{2}\right|_{1-g}=\alpha_{s} c_{2} M^{2}
$$

where $\alpha_{S} \approx 0.1$ at $1 \mathrm{TeV}, \mathrm{M}$ is a parameter of the technicolour theory of order $500 \mathrm{GeV}$ and $\mathrm{C}_{2}$ is the quadratic Casimir operator for colour $\mathrm{SU}(3)$, equal to $4 / 3$ for colour triplets and three for colour octets. The complete mass formula, to order $\alpha_{s}$ and $\alpha$, was given in Refs. 15) and 16); we will (formally at least) recover it from our methods in Section 5 .

In the 0 model, the breaking of SU(16) to $O(16)$ yields 135 Goldstone bosons. Of these, 63 have quantum numbers identical to those found in the SU case. Indeed, the breaking pattern of the SU case is neatly embedded inside that of the 0 case; we will make use of this feature in our analysis. The additional Goldstone bosons correspond to the 36 symmetric combinations of two technifermions and the similar combinations of two antifermions. These additional states include colour sextets $\left(\mathrm{U}_{\mathrm{a}} \mathrm{D}_{\mathrm{a}}\right)$, colour triplets $\left(\mathrm{U}_{\mathrm{a}} \mathrm{N}\right)$ and colour singlets $(E E)$. The coloured states acquire the large masses (2.7) from one gluon exchange. The new colour singlet states acquire masses of order $50 \mathrm{GeV}$ from $1-W$ exchange.

Both models contain a set of seven colour singlet bosons which do not receive large masses; in fact, these turn out to receive no mass at all to order $\alpha$ and $\alpha_{s}$. Of these, three are the fictitious Goldstone bosons eaten when the $W^{ \pm}$and $Z^{0}$ acquire masses; we will refer to these bosons as the $\pi^{a}$. The remaining four form an isotriplet and an isosinglet of light physical mesons. Eichten and Lane ${ }^{4)}$ have shown that these four light states appear in any model of several technifermion doublets which incorporates the Weinberg-Susskind mechanism; they have named these states $\mathrm{P}^{ \pm}, \mathrm{P}^{0}, \mathrm{P}^{\circ}$. These mesons are expected to acquire masses of order $10 \mathrm{GeV}$, from higher order electroweak effects, from possible interactions connecting techniquarks to technileptons 201,12$)$ and also, in a more realistic model incorporating ordinary quarks and leptons, from the interactions which connect these fermions to technifermions 4 , 10),12)*). These mesons are then light enough to be observed in the near future. It is possible that they are

*) Because of these last interactions, the isospin assignments and even the definite parity of the $P$ mesons should not be obvious from their masses and decays. 
light enough to be seen at PETRA and PEP; they are certainly light enough to be produced from the $Z^{0}$. The most direct applications of our formalism concern these particles. In Section 6 we will study their couplings to the $Z^{0}$; in our second paper ${ }^{14)}$ we will compute the order $\alpha^{2}$ contributions to their masses.

In this Section then we have set up a framework for analyzing models of technicolour and have recalled the particles whose properties we wish to understand. Let us now turn our attention to building a formalism to aid that understanding.

\section{THE SYMMETRIC CHIRAL LAGRANGIAN}

The next several Sections are devoted to the construction of the effective Lagrangian for a system of Goldstone bosons which may arise in a theory of technicolour. In doing this, we rely heavily on the work of Callan, Coleman, Wess and Zumino (CCWZ) ${ }^{7)}$, who worked out an elegant and general formalism yielding the effective Lagrangian for the Goldstone bosons formed in the spontaneous breaking of an arbitrary symmetry group $G$ to any subgroup $H$. Our purpose in these Sections will, essentially, be to review the analysis given by these authors and to adapt it to our more specific needs, although we will also introduce some useful notations. To complement their formal presentation, our explication of their work will be relatively heuristic.

The construction of this effective Lagrangian will proceed in three stages. The first is to write down a Lagrangian for the Goldstone bosons describing their technicolour interactions only; this Lagrangian should be symmetric under the chiral group' G. We will see in this Section that such a Lagrangian is constructed from a certain chiral-covariant derivative, which we will exhibit below. The second step is to couple the gauge bosons of weak interactions to the Goldstone bosons by gauging the generators of $G_{W}$; this we will discuss in section 4. These couplings explicitly break G; they require adding to the Lagrangian counterterms, involving Goldstone bosons fields alone, which transform non-trivially under G. These counterterms will be discussed in Section 5 .

The strategy of CCWZ is the following: in a theory of spontaneously broken symmetry, a multiplet of fields $\psi_{0}$, which transforms according to some irreducible representation of $G$, is in general broken into a number of pieces, each of which corresponds to an irreducible representation of $\mathrm{H}$. These pieces of $\psi_{0}$ represent particles with distinct masses and couplings; these particles need bear no obvious relation to one another. The full theory is symmetric under $G$, but we can make this symmetry apparent only if we implement it in such a way that the various irreducible representations of $H$ contained in $\psi_{0}$ are not mixed even by general $G$ transformations. These more general $G$ transformations should rather act on the broken symmetry vacuum, rotating the condensate which breaks $G$ down to $H$. 
Let us then introduce a field $I$ to represent the orientation of the symmetry breaking condensate in the group $G{ }^{*}$ ); it is useful to allow $\pi$ to vary in space-time. We will then factor a $G$ transformation into two pieces - a motion in $\mathrm{H}$ which rotates the $\mathrm{H}$ multiplets in $\psi_{0}$ and a motion in $\mathrm{G} / \mathrm{H}$ which rotates the local vacuum orientation $\Pi(\mathrm{x})$.

To make this strategy more precise, we introduce the following notation. (Again, our conventions are summarized in the Appendix.) Let the generators of $G$ be denoted by $G$ and generically labelled by indices from the beginning of the alphabet $(a, b, c)$. It will be useful to divide these into two classes: the unbroken generators, the generators of $\mathrm{H}$, denoted by $\mathrm{T}$ and labelled by indices from the middle of the alphabet $(i, j, k)$, and the broken generators denoted by $\mathrm{X}$ and labelled by indices from the end of the alphabet $(\mathrm{x}, \mathrm{y}, \mathrm{z}), \mathrm{G}, \mathrm{T}, \mathrm{X}$ will be used to refer both to the abstract generators and to the matrices which represent them; the relevant interpretation will be spelled out if it is not clear from the context.

Using this notation, we may represent the local orientation of the symmetry breaking condensate by the matrix

$$
\operatorname{expe}\left(i \pi_{y} x_{y}\right)
$$

If $\psi_{0}$ is a field which transforms according to a linear representation of $G$, we can recast it as

$$
\psi_{0}(x)=\exp _{\left(i \pi_{y}(x) X_{y}\right) \psi(x)}
$$

A G transformation of $\psi_{0}$ may be considered to act as follows

$$
\begin{aligned}
\psi_{0}^{\prime}(x) & =\exp \left(i \alpha_{a} G_{a}\right) \psi_{0} \\
& =\exp \left(i \alpha_{a} G_{a}\right) \exp \left(i \pi_{y}(x) X_{y}\right) \psi(x)
\end{aligned}
$$

*) We hope the reader will distinguish this use of $\Pi$ from the more specialized definition given in the previous Section. 


$$
=\exp \left(i J_{y}^{\prime}(x) X_{y}\right) \text { eyp }\left(i \mu_{i}(x) I_{i}\right) \psi(x), \stackrel{(3.3)}{\text { cont. }}
$$

where, in the last step, we have used the fact that a transformation in $G$ may always be factored into a transformation in $H$ times a transformation lifting one out of $H_{1} \Pi^{\prime}(x)$ and $\mu(x)$ are, in general, functions of $\Pi(x)$ and $\alpha$. The transformation of $\psi_{j}$ has thus been recast as a transformation

$$
\begin{gathered}
\Pi \longrightarrow \Pi^{\prime}(\pi, \alpha) \\
\psi \longrightarrow \psi^{\prime}=\exp \left(i \mu_{i}(\pi, \alpha) I_{i}\right) \psi,
\end{gathered}
$$

which realizes explicitly the factorization referred to above. The transformation (3.4) is a non-linear realization of $G$, which, it may be seen, becomes linear when one specializes to transformations in $\mathrm{H}$. CCWZ have shown that any nonlinear realization of $G$ which linearizes on $H$ may be brought into the form (3.4) by a suitable change of variables.

The transformation law (3.4a) implies that no function of the $\pi$ field, without derivatives, can be invariant under $G$; one may always set $\Pi^{\prime}$ to zero at any given point $x_{0}$ by choosing $\alpha=-\Pi\left(x_{0}\right)$ and performing the transformation (3.3). An invariant Lagrangian may thus only include terms with derivatives of I. A covariant derivative with a relatively simple transformation law under (3.4) was constructed by CCWZ; let us now recall that construction.

If $\psi_{0}$ transforms linearly under $G$, so does $\partial_{\mu} \psi_{0}$; this gives us a basis for forming covariant derivatives. Let us define functions $D(\pi)$ and $E(\pi)$ by

$$
\begin{aligned}
\operatorname{arfe} & \left(-i \Pi_{y} X_{y}\right) \partial_{\mu} \operatorname{erp}\left(i \pi_{y} X_{y}\right) \\
& =i \partial_{\mu} \Pi_{y}\left[D_{y z}(\pi) X_{z}+E_{y i}(\pi) T_{i}\right] .
\end{aligned}
$$

The left-hand side of (3.5) is a generator of $G$, so we have expanded it in terms of $x_{z}$ and $T_{i}$. Now we may use (3.5) to write $\partial_{\mu_{0}} \psi_{0}$ in terms of $I$ and $\psi$ : 


$$
\begin{aligned}
\partial_{\mu} \psi_{0}= & \partial_{\mu}\left[\operatorname{eyp}\left(i \pi_{y} x_{y}\right) \psi\right] \\
= & \operatorname{eyp}\left(i \pi_{y} x_{y}\right)\left[i \partial_{\mu} \pi_{y} D_{y z}(\pi) X_{z} \psi\right. \\
& \left.+\left(\partial_{\mu}+i \partial_{\mu} \pi_{y} E_{y i}(\pi) I_{i}\right) \psi\right] .
\end{aligned}
$$

Since $a_{\mu_{0}} \psi_{0}$ transforms under $G$ as does $\psi_{0}$, the object in square brackets has the transformation law (3.4b). However, since it is consistent to restrict $\psi$ to a given representation of $H$ and since, if this is done, the two objects in square brackets belong to different representations of $H$ in the same representation of $G$, these two objects must transform independently according to (3.4b). If we define

$$
\begin{gathered}
D_{\mu} \psi=\left(\partial_{\mu}+i \partial_{\mu} \Pi_{y} E_{y i} T_{i}\right) \psi \\
D_{\mu} \Pi_{z}=\partial_{\mu} \Pi_{y} D_{y z},
\end{gathered}
$$

these fields must transform under $G$ according to

$$
\begin{aligned}
& D_{\mu} \psi \rightarrow \operatorname{eyp}\left(i \mu_{i}(\pi, \alpha) T_{i}\right) D_{\mu} \psi \\
& D_{\mu} \pi_{y} \rightarrow\left[\exp \left(i \mu_{i}(\pi, \alpha) T_{i}\right)\right]_{y z} D_{\mu} \pi_{z},
\end{aligned}
$$

where, in the second line, $T_{i}$ is a representation matrix in the representation of $\mathrm{H}$ to which the $\mathrm{X}_{\mathrm{y}}$ belong. The transformation laws (3.4b) and (3.8) imply that a function of $\psi, D_{\mu} \psi$, and $D_{\mu} \Pi$ is invariant under general $G$ transformations if it is superficially invariant under $H$ transformations. This is a most simple prescription for constructing the terms of an effective Lagrangian.

We are interested here in the interactions of Goldstone bosons alone; thus we will construct our effective Lagrangian from the field $\pi$ or rather from its covariant derivative $D_{\mu}$ ! only. Since this Lagrangian is meant to be a phenomenological description of an underlying, more fundamental, theory of strong interactions, we should include every possible G invariant interaction, each appearing with an arbitrary coeffecient. These coefficients generally carry mass dimensions; since the field $\Pi$ is dimensionless, a term in the Lagrange 
density with $n$ derivatives has a coefficient of dimension (mass) ${ }^{4-n}$. The scale for these factors of mass should be set by the natural scale of the theory; in our case, this is $M_{T C}$. However, this means that, for a process occurring at energy $E \ll M_{T C}$, each extra derivative gives a suppression factor $E / M_{T C} 9$ ). To make a phenomenological description of such low-energy processes, it suffices to keep only the term with the fewest derivatives:

$$
\mathscr{L}=\frac{1}{2} f_{\pi}^{2}\left(D_{\mu} \pi_{x}\right)^{2}
$$

The constant $f_{\pi}$, with dimensions of mass, will later be identified with the pion decay constant. In introducing only one such constant, we have used our assumption that the $\mathrm{X}_{\mathrm{z}}$ comprise a single irreducible representation.

Actually, it is useful at this point to introduce the whole symmetric space structure of symmetry breaking, in particular, the parity invariance (2.1). This allows (3.9) to be cast into a more explicit, but equally simple, form: $D_{y z}(I)$, defined in (3.5), may be computed from the identity

$$
\begin{aligned}
& \text { exp }(-i \pi \cdot X) \partial_{\mu} \text { exp }(i \pi \cdot X) \\
& =\sum_{n=0}^{\infty} \frac{i^{n+1}}{(n+1) !}\left[\cdots\left[\left[\partial_{\mu} \pi \cdot X, \pi \cdot X\right], \pi \cdot X\right], \cdots \pi_{1}{ }_{2}^{13 \cdot 10}\right]^{.}
\end{aligned}
$$

Let us define $t_{a b c}$ by

$$
\left[G_{a}, G_{b}\right]=t_{a b c} G_{c} \text {, }
$$

and denote

$$
t_{a \times b} \Pi_{x}=(t \cdot \pi)_{a b}
$$

These matrices represent the (broken) generators of $G$ in the adjoint representation; they are Hermitian and antisymmetric. The right-hand side of (3.10) may now be rewritten as

$$
\sum_{n=0}^{\infty} \frac{i^{n+1}}{(n+1) !} \partial_{\mu} \Pi_{x}(t \cdot \pi)_{x Q}^{n} G_{a}
$$


However, each multiplication by $\Pi \cdot X$, and thus each application of $t \cdot \pi$, flips the parity: the terms in (3.13) for which $G_{a}$ is an $x$, are precisely those for which $\mathrm{n}$ is even. Then, comparing (3.13) to (3.5), we find

$$
D_{y z}(\pi)=\sum_{m=0}^{\infty} \frac{(-1)^{m}}{(2 m+1) !}(t \cdot \pi)_{y z}^{2 m}=\left(\frac{\sin t \cdot \pi}{t \cdot \pi}\right)_{y z} .
$$

In this notation, (3.9) becomes

$$
\mathcal{L}=\frac{1}{2} f_{\pi}^{2} \partial_{\mu} \pi_{x}\left(\frac{1-\cos (2 t \cdot \pi)}{2(t \cdot \pi)^{2}}\right)_{x y} \partial^{\mu} \Pi_{y} .
$$

One may derive Feynman rules from (3.15) by rescaling the field

$$
\pi_{x}=f_{\pi} \Pi_{x}
$$

and expanding in powers of $\pi$

$$
\begin{gathered}
\mathcal{L}=\frac{1}{2}\left(\partial_{\mu} \pi_{x}\right)^{2}+\frac{1}{6 f_{n}^{2}}\left(t_{a x y} \pi_{x} \partial_{\mu} \pi_{y}\right)^{2} \\
+\cdots .
\end{gathered}
$$

The mass term for $\pi$ has been forbidden by $G$ invariance; $\pi$ is the Goldstone boson field. The interactions of the Goldstone bosons are, at this level, specified in terms of the single parameter $\mathrm{f} \pi$.

\section{GAUGING THE CHIRAL LAGRANGIAN}

In the previous Section we discussed the phenomenology of the interactions of Goldstone bosons in a strong interaction theory treated in isolation from perturbing interactions. One of the crucial features of technicolour interactions, however, is their relation to the weak interactions and to other gauge symmetries which couple weakly to technifermions. To discuss this relation, we will introduce the gauge mesons of the group $G_{w}$ into the chiral Lagrangian. In the formation of CCWZ, this is straightforwardly accomplished. We will devote the first half of this Section to the general formalism for these gauge couplings; then we will work out some of these couplings in the specific models introduced in Section 2 .

The symmetry implemented in Section 3 was a global symmetry. We would now like to promote a subgroup $G_{W}$ of the global group $G$ to a local symmetry. 
The local invariance requires that the ordinary derivative of $\psi_{0}$ be replaced by a covariant derivative which we may write

$$
\underline{D}_{\mu} \psi_{0}=\left(\partial_{\mu}-i A_{\mu}^{A} G_{A}\right) \psi_{0} \text {. }
$$

In (4.1), the $\mathrm{G}_{\mathrm{A}}$ are generators of $\mathrm{G}$ defined to include coupling constants and other arbitrary normalization factors. We will use capital letters as indices to represent such arbitrarily, as opposed to conventionally, normalized generators. It is also convenient to generalize the structure constants tabc to allow them to share the normalization of the $G_{A}$. Denote

$$
\left[G_{A}, G_{b}\right]=t_{A b c} G_{c}, t_{A \times b} \pi_{x}=(t \cdot \pi)_{A b},
$$

and define $t_{a b c}$ by insisting that $t$ be totally antisymmetric under interchange of indices.

The local symmetry $G_{w}$ is, of course, realized linearly on $\psi_{0}$. Using (3.2), however, we may recast the realization of $G_{w}$ as a non-linear realization of the form (3.4). By rewriting (4.1) in terms of $\Pi$ and $\psi$, we can form derivatives which are both gauge and chiral covariant, with local transformation laws of the form (3.8).

To accomplish this, we must split the generators $G_{A}$ into unbroken and broken pieces"). In the presence of the $\Pi$ field, this separation is given by functions $F(\Pi), H(\Pi)$ defined by

$$
\operatorname{exf}(-i \pi \cdot x) G_{A} \text { eyfe }(i \pi \cdot x)=F_{A y}(\pi) X_{y}+H_{A i}(\pi) T_{i(i, 3)}
$$

Then we can rewrite the covariant derivative of $\psi_{0}$ in the form

$$
\begin{aligned}
\underline{D}_{\mu} \psi_{0}= & \exp (i \pi \cdot x)\left[\left(i D_{\mu} \pi_{y}-i A_{\mu}^{A} F_{A y}\right) x_{y} \psi\right. \\
& \left.+\left(D_{\mu}-i A_{\mu}^{A} H_{A i} T_{i}\right) \psi\right] .
\end{aligned}
$$

*) We assume that the alignment of $G$ relative to the symmetry breaking has already been correctly determined 15$), 16)$. 
We can read from this equation covariant derivatives having (3.8) as local transformation laws:

$$
\begin{aligned}
& \theta_{\mu} \psi=\left[\partial_{\mu}+i\left(\partial_{\mu} \pi_{y} E_{y i}-A_{\mu}^{A} H_{A i}\right) T_{i}\right] \psi \cdot{ }_{(4.5)} \\
& \partial_{\mu} \pi_{y}=\partial_{\mu} \Pi_{x} D_{x y}-A_{\mu}^{A} F_{A y} .
\end{aligned}
$$

The generalization of (3.9), including the couplings of Goldstone bosons to gauge mesons of $G_{W}$, is then

$$
\mathcal{L}=\frac{1}{2} f_{\pi}^{2}\left(D_{\mu} \pi_{y}\right)^{2}
$$

As we did with (3.9), we can use the parity invariance (2.1) to produce a more explicit form of (4.6). Recall the identity

$$
\begin{aligned}
& \exp (-i \pi \cdot x) G_{A} \exp (i \pi \cdot x) \\
& =\sum_{n=0}^{\infty} \frac{i^{n}}{n !}\left[\cdots\left[\left[G_{A}, \pi \cdot x\right], \pi \cdot x\right], \cdots \cdot \frac{\pi}{2} \cdot x\right] \\
& =\sum_{n=0}^{\infty} \frac{i^{n}}{n !}(t \cdot \pi)_{A b}^{n} G_{b} .
\end{aligned}
$$

(2.1) implies that if $G_{A}$ in (4.7) is a $T, G_{b}$ will be a $T$ when $n$ is even and an $\mathrm{X}$ when $\mathrm{n}$ is odd. One may, in this way, recognize

$$
\begin{aligned}
& F_{I X}=i(\sin t \cdot \pi)_{I x}, \\
& F_{Z x}=(\cos t \cdot \pi)_{Z x} .
\end{aligned}
$$

Let us denote the direct decomposition of each $G_{A}$ into $T_{i}$ and $X_{y}$ by

$$
G_{A}=T_{I(A)}+X_{Z(A)}
$$

we will of ten use only the indices $I(A), Z(A)$ to refer to these arbitrarily normalized generators. Using this notation, we may rewrite

$$
\theta_{\mu} \Pi_{y}=\partial_{\mu} \Pi_{x} \cdot\left(\frac{\sin t \cdot \pi}{t \cdot \pi}\right)_{x y}-
$$




$$
-A_{\mu}^{A}\left[(\cos t \cdot \pi)_{Z(A) y}+i(\sin t \cdot \pi)_{I(A) y}\right] \cdot \begin{aligned}
& (4.10) \\
& \text { cont. }
\end{aligned}
$$

Inserting this formula into (4.6), and taking care that $t \cdot \Pi$ is antisymmetric, we recover as the final form of the chiral Lagrangian

$$
\begin{aligned}
& \mathcal{L}=\frac{1}{2} f_{\pi}^{2}\left[\partial_{\mu} \Pi_{x}\left(\frac{1-\cos (2 t \cdot \pi)}{2(t \cdot \pi)^{2}}\right)_{x y} \partial^{\mu} \Pi_{y}\right. \\
& -\partial^{\mu} \Pi_{x} A_{\mu}^{A}\left\{\left(\frac{\sin (2 t \cdot \pi)}{t \cdot \pi}\right)_{x Z(A)}-i\left(\frac{1-\cot (2 t \cdot \pi)}{t \cdot \pi}\right)_{x J(\mathcal{A})}\right\}^{(4.11)} \\
& \begin{array}{c}
+A_{\mu}^{A}\left\{\left(\frac{1+\cos (2 t \cdot \pi)}{2}\right)_{Z(A) Z(B)}+\left(\frac{1-\cos (2 t \cdot \pi)}{2}\right)_{I(A) J(B)}\right. \\
\left.+i(\sin (2 t \cdot \Pi))_{J(A) Z(B)}\right\} A^{\mu B}
\end{array}
\end{aligned}
$$

Note that the definite parity of the Goldstone bosons is made completely explicit in this formula: gauge bosons which couple to even-parity currents can produce only an even number of Goldstone bosons; gauge bosons which couple to odd-parity currents can produce only an odd number of Goldstone bosons. This is a most convenient feature of the particular realization chosen by CCWZ.

To give some insight into the physics contained in (4.11), let us work out some of the vertices which this Lagrangian implies for the two illustrative models set up in Section 2. We should first introduce a few more group theoretic conventions: label generators of $\mathrm{SU}(8)$ by $\mathrm{g}_{\alpha}$ and normalize them to

$$
\operatorname{tr}\left(g_{\alpha} g_{\beta}\right)=\frac{1}{2} \delta_{\alpha \beta} \text {. }
$$

Normalize generators of $\mathrm{SU}(16)$ to

$$
\operatorname{tr}\left(G_{a} G_{b}\right)=\delta_{a b}
$$

The symmetry group $\mathrm{SU}(8) \times \mathrm{SU}(8) \times \mathrm{U}(1)$ of the $\mathrm{SU}$ model fits neatly inside $\mathrm{SU}(16)$ in the manner indicated by (2.4). The $\mathrm{SU}(8) \times \mathrm{SU}(8)$ matrices corresponding to vector and axial vector transformations are respectively 


$$
T=\left(\begin{array}{c|c}
g & 0 \\
\hline 0 & -g^{T}
\end{array}\right), \quad x=\left(\begin{array}{c|c}
g & 0 \\
\hline 0 & g^{T}
\end{array}\right) .
$$

We can identify the vector and axial vector currents of the chiral Lagrangian by imagining that the full chiral symmetry $G$ had been gauged and reading from (4.1I) the coefficients of $A_{\mu}^{k}$ and $A_{\mu}^{z}$. This yields, for the axial vector currents,

$$
J_{z}^{\mu}=f_{\pi}^{2} \partial_{\mu} \Pi_{x}\left(\frac{\sin (2 t \cdot \pi)}{2 t \cdot \pi}\right)_{x z} .
$$

Normalizing the physical field as in (3.16), we can see that (at the level of tree graphs)

$$
\left\langle\Omega\left|J_{z}^{\mu}\right| \pi_{x}(z)\right\rangle=-i f_{\pi} f^{\mu} \delta_{x z} .
$$

Thus, $f_{\pi}$ is the pion decay constant, normalized in a manner that, in the usual strong interactions, would correspond to $f_{\pi}=90 \mathrm{MeV}$.

Let us now note some aspects of the structure of the chiral Lagrangian (4.11) which would be derived from the $G_{W}$ interactions presented in (2.5). It will be useful to divide the gauge couplings into broken and unbroken pieces. The colour gluons couple only to unbroken generators. For $W_{\mu}^{\mathrm{a}}$ and $B_{\mu}$, this separation reads

$$
\begin{aligned}
A_{\mu}^{A} T_{I(A)} & =\frac{1}{2}\left(g W_{\mu}^{a}+g^{\prime} \delta^{a 3} B_{\mu}\right)\left(\begin{array}{l|l|l}
\tau_{a} \otimes 1_{4} & \\
\hline & -\tau_{a}^{T} \otimes I_{4}
\end{array}\right) \\
& +\frac{1}{2} g^{\prime} B_{\mu}\left(\begin{array}{ll|l}
1_{2} \otimes B & \\
\hline & -1_{2} \otimes B
\end{array}\right),
\end{aligned}
$$

$$
A_{\mu}^{A} X_{Z(A)}=\frac{1}{2}\left(g W_{\mu}^{a}-g^{\prime} \sigma^{a{ }^{3} B_{\mu}}\right)\left(\frac{\tau_{a} \otimes 1_{4} \mid}{\mid \tau_{a}^{T} \otimes 1_{4}}\right) !^{(4.18)}
$$

A first obvious feature of (4.11) is the presence of a term quadratic in $A_{\mu}^{Z(A)}$ and containing no $\Pi$ fields. This is a mass term for the gauge bosons corresponding to broken generators of $\mathrm{G}_{\mathrm{W}}$, just the one required by the Higgs mechanism. Explicitly, this term is 
$-18-$

$$
\begin{aligned}
\frac{1}{2} f_{\pi}^{2} A_{\mu}^{A} & (1)_{Z(A) Z(B)} A^{\mu B} \\
& =\frac{1}{2} f_{\pi}^{2} t_{2}\left(A_{\mu}^{A} X_{Z(A)} A^{\mu B} X_{Z(B)}\right) \\
& =\frac{1}{2}\left(\frac{f_{\pi}}{2}\right)^{2}\left(g W_{\mu}^{2}-g^{\prime} \delta^{a 3} B_{\mu}\right)^{2} \cdot 4
\end{aligned}
$$

[The definition of $(1) \mathrm{Z}(\mathrm{A}) \mathrm{Z}(\mathrm{B})$ indicated compensates (4.13) for the arbitrary normalization of the generators $X_{Z(A)}$ - T. This term yields precisely the masses for $W^{ \pm}$and $Z^{0}$ found by Weinberg and Susskind ${ }^{3)}$

$$
m_{w}^{2}=L\left(\frac{f_{\pi} g}{2}\right)^{2}, \quad m_{z}=m_{w} \mid \cos \theta_{w}, \text { (4.20) }
$$

where $L$ is the number of weak doublets of technifermions, $L=4$ in our models. (4.20) gives the value of $f_{\pi}$ for technicolour; if $L=4$,

$$
f_{\pi}=120 \mathrm{GeV}
$$

In addition to this mass term, one has in the second term of (4.11) the usual coupling

$$
-f_{\pi} \partial_{\mu} \pi_{y} t_{r}\left(X_{y} X_{z(A)}\right) A_{\mu}^{A}
$$

of the gauge bosons (which receive mass) to the Goldstone bosons which they will eat. It is convenient to remove this coupling by working in the Landau gauge.

By parity invariance, only the vector parts of the gauge boson currents can create pairs of Goldstone bosons. Since these vector currents generate unbroken symmetries, the couplings of gauge bosons to pairs of Goldstone bosons must be proportional to their good quantum numbers. To see how this arises in our formalism, let us write out the $A-\pi-\pi$ interaction term from the second line of (4.11), incorporating the rescaling (3.16):

$$
\begin{aligned}
\mathcal{L}_{\pi \pi A} & =i \partial_{\mu} \pi_{x}(t \cdot \pi)_{x j(A)} A^{\mu A} \\
& =i \partial_{\mu} \pi_{x} \pi_{y} t_{x y j(A)} A^{\mu A}
\end{aligned}
$$




$$
=-i \partial_{\mu} \pi_{x} \pi_{y} \operatorname{tr}\left(X_{x}\left[T_{1(A)}, X_{y}\right]\right) A^{\mu A} \cdot{ }^{(4.23)} \text { cont. }
$$

It is instructive to work out explicitly the couplings of the physical photon and $Z^{0}$ fields to $\pi$ 's. To do this, rewrite the terms involving $W_{\mu}^{3}$ and $B_{\mu}$ in (4.17) and (4.18) in terms of $A_{\mu}$ and $Z_{\mu}$ :

$$
\begin{aligned}
& A_{\mu}^{A} T_{I(A)}=\operatorname{eA} A_{\mu}\left(\begin{array}{l|l}
Q & 0 \\
\hline 0 & -Q
\end{array}\right) \\
& +\frac{e}{\sin 2 \theta_{W}} Z_{\mu}\left(\frac{\tau_{3} \otimes t_{4}-2 \sin ^{2} \theta_{W} Q}{} \mid \frac{-\tau_{3} \otimes 1_{4}+2 \sin ^{2} \theta_{W} Q}{\mid}\right), \\
& A_{\mu}^{A} X_{Z(A)}=\frac{e}{\sin 2 \theta_{W}} Z_{\mu}\left(\begin{array}{l|l}
\tau_{3} \otimes 1_{4} & \\
\hline & \tau_{3} \otimes I_{4}
\end{array}\right) \text {, }
\end{aligned}
$$

where $\underline{Q}=\left(\tau_{3} \times I_{4}+1 / 2 I_{2} \times B\right)$ is the electric charge matrix, whose eigenvalues are $Q=\left(I_{3}+B / 2\right)$. Choosing $x_{y}$ as eignevalues of $\underline{Q}$ and then inserting (4.24) into (4.23), one can read off directly the Feynman rules for the $A$ and $Z^{0}$ couplings; these are given in Fig. 1.

Couplings of two vector bosons to one Goldstone boson arise only from the last term of (4.11). The form of the interaction is

$$
\mathcal{L}_{\pi \pi A}=i f_{\pi} t_{I(A) \times Z(B)} \pi_{x} A_{\mu}^{A} A^{\mu B} \text {. }
$$

This vertex is most severely restricted: it exists only if the Goldstone boson corresponds to a generator of $G$ which is included in the Lie algebra of $G_{W}$. This, in turn, requires that the Goldstone boson appearing in (4.26) is a fictitious one. The chiral Lagrangian has no vertices coupling two vector bosons to a physical Goldstone boson; this feature will enter our discussion in Section 6 .

We complete this Section by displaying one more set of vertices which will be useful to us: the "seagull" vertices involving two $\pi^{\prime} s$ and two gauge bosons. Interactions of this type arise from two terms of (4.11): 


$$
\begin{aligned}
& \mathcal{L}_{\pi \pi A A}=\frac{1}{2} f_{\pi}^{2} A_{\mu}^{A} A^{\mu B}\left[-(t \cdot \Pi)_{Z(A) Z(B)}^{2}+(t \cdot \Pi)_{I(A) J(B)}^{2}\right] \\
& =\frac{1}{2} A_{\mu}^{\wedge} A^{\mu B} \pi_{x} \pi_{y} x \\
& x \quad\left(\operatorname{tr}\left\{x_{x}\left[T_{I(A)},\left[T_{I(B)}, x_{y}\right]\right]\right\}\right. \\
& \left.-\operatorname{tr}\left\{X_{x}\left[X_{z(A)},\left[X_{z(B)}, X_{y}\right]\right]\right\}\right) \text {. }
\end{aligned}
$$

The factor in parentheses is straightforward to work out explicitly, at least for the Goldstone bosons of the SU model, which correspond to $\mathrm{x}_{\mathrm{y}}$ of the diagonal form (4.14). For the photon, the second term in parentheses simply vanishes. For the $W$ bosons, and for diagonal $X$, one can see that the two terms in parentheses cancel. For the $2^{0}$, this cancellation is incomplete; the interaction term, for diagonal $\mathrm{x}_{\mathrm{y}}$ becomes, using (4.25) and (4.26) in (4.27):

$$
\begin{gathered}
\mathcal{L}_{\pi \pi z Z}=\frac{1}{2} Z_{\mu} Z^{\mu} \pi_{x} \pi_{x}\left(\frac{e}{\sin 2 \theta_{W}}\right)^{2} \times \\
\times\left[\left(I_{3}-2 \sin ^{2} \theta_{W} Q\right)^{2}-\left(I_{3}\right)^{2}\right] \\
=\frac{1}{2} Z_{\mu} Z^{\mu} \pi_{x} \pi_{x} \frac{e^{2} Q}{\cos ^{2} \theta_{W}}\left(-I_{3}+\sin ^{2} \theta_{W} Q\right) .
\end{gathered}
$$

The full set of two gauge boson vertices is shown in Fig. 1.

\section{MASS COUNTERTERM FOR THE CHIRAL LAGRANGIAN}

The simplicity of the chiral Lagrangian (3.15) resulted from the constraint of $G$ invariance. In the previous Section, however, we added to (3.15) new terms - the couplings of Goldstone bosons to the gauge bosons of $G_{W}$ - which do not respect this invariance. These couplings are weak, but we expect nevertheless that they should induce modifications of the interactions of Goldstone bosons with one another. The most obvious such modification is the generation of masses, of order $\mathrm{eM}_{\mathrm{TC}}$ or $\mathrm{g}_{\mathrm{S}} \mathrm{M}_{\mathrm{TC}}$, for pseudo-Goldstone bosons. However, we will see that new non-linear couplings also appear. These couplings include an explicit 
suppression factor of $\alpha$ or $\alpha_{S}$, but they need not contain derivatives, so that they lack the soft-pion suppression of the vertices of the symmetric Lagrangian. For a process at energy $E$, the vertices of (3.17) are suppressed by a factor $\left(E / f{ }^{2}\right)^{2}$; when $E$ is of the order of a $W^{ \pm}, Z^{0}$ or pseudo-Goldstone mass, this factor is comparable to $\alpha$ or $\alpha_{S}$. The induced. $G$ violating terms may then be as important as the terms of the symmetric Lagrangian for interesting processes; it is important that we identify and understand them. We will see in this section that the Goldstone boson interactions induced by the virtual exchange of one $G_{w}$ boson may be summarized as a single function of $\pi$. The coefficient of this new term introduces into the phenomenological Lagrangian one new dimensionful parameter.

To demonstrate that some term has been omitted from the formalism we have constructed thus far, we can identify, in the perturbation theory derived from (4.11), divergent graphs which need renormalization counterterms of a new form. We might, for example, compute the correction to the $\pi$ propagator which results from the exchange of one $G_{w}$ boson; in Landau gauge, this is given by the graphs of Fig. 2a ${ }^{*}$. The vertices which appear in these graphs are given in (4.23) and (4.27). In Landau gauge, the first graph vanishes as $p \rightarrow 0$; the second graph, however, gives a contribution to the $\pi$ masses in the form of a quadratically divergent integral times the group theory factor

$$
\begin{aligned}
& \sum_{A}\left(t r\left\{x_{x}\left[T_{I(A)},\left[T_{I(A)}, x_{y}\right]\right]\right\}\right. \\
&-\left.t r\left\{x_{x} \cdot\left[x_{z(A)},\left[x_{z(A)}, x_{y}\right]\right]\right\}\right) .
\end{aligned}
$$

One can find additional quadratically divergent diagrams by examining radiative

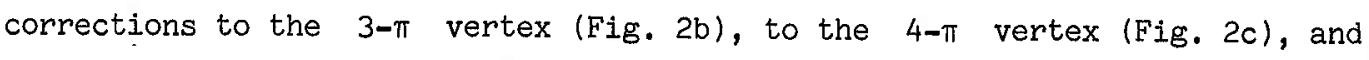
so forth. We need to find a counterterm which reproduces the form of the mass matrix (5.1) and also the forms of these new interactions. (We postpone a discussion of the logarithmic divergences of chiral perturbation theory to our second paper ${ }^{14)}$.) What constrains the form of possible counterterms? A virtual $\mathrm{G}_{\mathrm{w}}$ boson couples to Goldstone bosons via a product of two currents, an object transforming under $G$ according to the, in general, reducible (adjoint) $x$ $x$ (adjoint) representation. Thus, the set of Goldstone boson interactions induced by virtual one vector boson exchange, must have the form of a function of the fields $\Pi_{x}$ which transforms linearly, as a component of the (adjoint) $x$ $x$ (adjoint) representation of $G$, when the $\Pi_{x}$ undergo the non-linear transformation (3.4a).

\footnotetext{
*) In other gauges, there are additional graphs involving the fictitious Goldstone bosons.
} 
This problem is a special case of the following problem: to construct a function of fields with the tranformation laws (3.4) which transforms linearly according to a given representation $R$ of $G$. CCWZ gave a solution to this more general problem by reversing the procedure of factorization used in Section 3 . If we imagine breaking $G$ down to $H$, the representation $R$ breaks up into irreducible representations of $\mathrm{H}$; call one of these $r$. Let $\psi$ be a field which transforms linearly under $H$ according to $r$. Then, if $\Pi, \psi$ are given the transformation laws (3.4) under $G$, a combination $f_{R}$ of these fields which transforms linearly under $G$ according to $R$ is given by

$$
f_{R}(\pi, \psi)=\exp (i \pi \cdot x) \psi
$$

In (5.2), $x_{y}$ is a representation matrix of the representation $R$. One may build a field belonging to $R$ from the $\Pi_{y}$ alone if $R$ contains an invariant under $\mathrm{H}$; in that case, one may replace the dynamical field $\psi$ by the invariant $I$ and write

$$
f_{R}(\pi)=\exp (i \pi \cdot x) I
$$

CCWZ proved that this is the only way to construct an object transforming linearly under $G$ from the chiral fields.

Let us now attempt to construct terms transforming according to the (adjoint) $x$ $\times$ (adjoint) representation of $G$, under the symmetric space restrictions set out below (2.1). In this case, the adjoint representation of $G$ breaks up into two irreducible representations - the adjoint representation of $H$ and the representation of $\mathrm{H}$ to which the $\mathrm{x}_{\mathrm{y}}$ belong. This means that the (adjoint) $x$ $x$ (adjoint) representation of $G$ contains two $H$ invariants, $\delta_{i j}$ and $\delta_{x y}$. However, this representation of $G$ is not irreducible and, in particular, it contains a $G$ invariant $\delta_{a b}=\delta_{i j}+\delta_{x y}$. Using this $G$ invariant for $I$ in (5.3) would yield a trivial $f_{R}(I)$; this leaves only one possible functional form for the required counterterm.

To see how this works more explicitly, let us represent an element of the (adjoint) $\times$ (adjoint) representation of $G$ as a matrix with two indices. Setting $I=\delta_{i j}$ and writing out (5.3), using (3.12) to represent the matrices $\pi \cdot x$, we have

$$
\begin{aligned}
f_{a b}(\pi) & =[\exp (i t \cdot \pi)]_{a i}[\exp (i t \cdot \pi)]_{b j} \delta_{i j} \\
& =[\exp (i t \cdot \pi)]_{a j}[\exp (-i t \cdot \pi)]_{j b} .
\end{aligned}
$$


$-23-$

Replacing $\delta_{i j}$ by $\delta_{a b}$ gives a triviality

$$
[\exp (i t \cdot \pi)]_{a c}[\exp (-i t \cdot \pi)]_{c b}=\delta_{a b} \text {. }
$$

so, indeed, (5.4) is the only possible form from which a counterterm may be built.

We can simplify (5.4) somewhat by using the constraints of (2.1). Allowing the indices of $f(I I)$ to represent gauged generators and then using the index. notation (4.9), we can write $(5.4)$ as

$$
\begin{aligned}
& f_{A B}(\pi)=\left[(\cos t \cdot \pi)_{I(A) j}+i(\sin t \cdot \pi)_{z(A) j}\right] x \\
& x \quad\left[(\cos t \cdot \pi)_{j g(B)}-i(\sin t \cdot \pi) j z(B)\right] \\
& =\frac{1}{2}\left[(\cos (2 t \cdot \pi))_{I(A) I(B)}-(\cot (2 t \cdot \pi))_{Z(A) Z(B)}\right. \\
& +i(\sin (2 t \cdot \pi))_{Z(A) I(B)}-i(\sin (2 t \cdot \pi))_{I(A) Z(B)}
\end{aligned}
$$

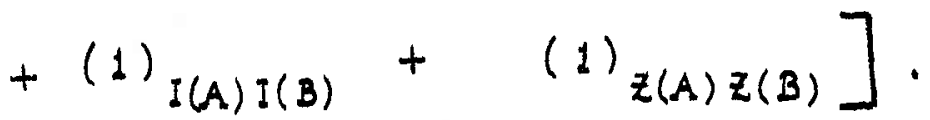

Dropping the last two terms, which are independent of $\Pi$, and recalling the constrains of parity, we may abbreviate this as

$$
\left.f_{A B}(\Pi)=\frac{1}{2}[\exp (2 i t \cdot \Pi)]_{(I(A)+Z(A))(I(B)-Z(B))}\right)^{(5.7)}
$$

To form the particular counterterm we need, we should weight the functions $f_{a b}(I)$ corresponding to products of currents with the $G_{w}$ coupling constants associated with those currents. This is automatically done by using the generators normalized as in (4.1). The counterterm is then

$$
\delta \mathcal{L}=\frac{f_{\pi}^{2} M^{2}}{16 \pi} \sum_{A}[\operatorname{exz}(2 i t \cdot \pi)]_{(I(A)+Z(A))(J(A)-Z(A))} \cdot(5.8)
$$

We have assigned this term a coefficient of dimension (mass) ${ }^{4}$, introducing a new parameter $M$ with dimensions of mass. To identify this parameter, let us examine the piece of (5.8) quadratic in $\pi$ fields

$$
\delta L_{\pi \pi}=-\frac{1}{2} \frac{M^{2}}{4 \pi} \sum_{A}\left[(t \cdot \pi)^{2} I(A) I(A)-(t \cdot \pi)^{2} Z(A) Z(A)\right] ;(5.9)
$$


that is,

$$
\delta \mathcal{L}_{\pi \pi}=-\frac{1}{2} m_{x y}^{2} \pi_{x} \pi_{y}
$$

with

$$
\begin{aligned}
m_{x y}^{2}=\frac{M^{2}}{4 \pi} & \sum_{A}\left(\operatorname{tr}\left\{X_{x}\left[T_{I(A)},\left[T_{I(A)}, X_{y}\right]\right]\right\}\right. \\
& \left.-\operatorname{tr}\left\{X_{x}\left[X_{z(A)},\left[X_{z(A)}, X_{y}\right]\right]\right\}\right) .
\end{aligned}
$$

(5.11) has the structure (5.1), so (5.8) is indeed a counterterm for the graphs of Fig. 2a. However, more importantly, (5.11) agrees precisely with the expression for the mass matrix of pseudo-Goldstone bosons, which had been derived previously using current algebra. [See, in particular, Eq. (2.30) of Ref. 15).] This allows us to identify the parameter $M$ with a parameter of current algebra introduced in Ref. 15) and estimated there, at least for technifermions in the fundamental representation of an $S U(N)$ technicolour group, to have a value

$$
M \cong 960 \mathrm{GeV} / \sqrt{N} \text {. }
$$

For later convenience, let us display the cubic and quartic terms of (5.8). The cubic terms are

$$
\delta \mathcal{L}_{3 \pi}=-i \frac{f_{\pi}^{2} M^{2}}{12 \pi} \sum_{A}\left[(t \cdot \pi)^{3} Z(A) I(A)-(t \cdot \pi)^{3} I(A) Z(A)\right](5.13)
$$

or, using the antisymmetry of $t \cdot \Pi$,

$$
\delta \mathcal{L}_{3 \pi}=i \frac{M^{2}}{6 \pi f_{\pi}} \sum_{A}(t \cdot \pi)^{3} I(A) Z(A) .
$$

Similarly, the quartic terms may be reduced to

$$
\delta \mathcal{L}_{4 \pi}=\frac{M^{2}}{24 \pi f_{\pi}^{2}} \sum_{\Lambda}\left[(t \cdot \pi)_{I(A) J(A)}^{4}-(t \cdot \pi)_{Z(A) Z(A)}^{4}\right] .
$$

We should remark that (5.8) apparently also contains a term linear in $\pi$ :

$$
\delta f_{\pi}=-i \frac{f_{\pi} M^{2}}{4 \pi} \sum_{A}(t \cdot \pi)_{I(A) Z(A)} .
$$

However, if we have chosen the correct orientation of the vacuum condensate, a $\pi$ cannot disappear into the vacuum without the intervention of a source; thus, (5.16) must be required to vanish. This gives a stability condition on the vacuum orientation, 


$$
0=\sum_{A}\left(t_{y}\right)_{I(A) Z(\Lambda)}=\sum_{A} \operatorname{tr}\left(X_{y}\left[T_{I(A)}, X_{z(A)}\right]\right)^{(5.17)}
$$

identical to the one found from current algebra [Eq. (2.25) of Ref. 15)]

To complete our examination of $(5.8)$, let us specialize it to the case $G=S U(N) \times S U(N)$, broken to $H=S U(N)$. If we normalize the $S U(N)$ generators according to (4.12) and construct the generators of $G$ as shown in (4.14), the structure constants of $G$ are simply equal to $S U(N)$ structure constants ${ }_{\alpha \beta \gamma}$, given by

$$
\left[g_{\alpha}, g_{\beta}\right]=t_{\alpha \beta \gamma} g_{\gamma},(t \cdot \pi)_{\alpha \gamma}=t_{\alpha \beta \gamma} \pi_{\beta} .
$$

Let us label the diagonal blocks of the gauged generators $G_{A}$

$$
G_{A}=\left(\begin{array}{c|c}
g_{L(A)} & 0 \\
\hline 0 & -\left(g_{R(A)}\right)^{T}
\end{array}\right),
$$

$\mathrm{g}_{\mathrm{L}(\mathrm{A})}$ and $\mathrm{g}_{\mathrm{R}(\mathrm{A})}$ are the matrices which appear in the left- and right-handed couplings of the gauge boson $\dot{A}_{\mu}^{A}$. Then $(5.8)$ can be written in terms of the $\mathrm{SU}(\mathrm{N})$ structure constants as follows

$$
\delta \mathcal{L}=\frac{f_{\pi}^{2} M^{2}}{16 \pi} \sum_{A}[\exp (2 i t \cdot \pi)]_{L(A) R(A)} .
$$

It was shown in Refs. 15) and 16) that for this symmetry breaking pattern, only gauge bosons with both left and right-handed couplings can give mass to Goldstone bosons. We see from (5.20) that this conclusion extends to the full set of $G$ violating interactions induced by one gauge boson exchange.

\section{TECHNION PRODUCTION AT THE $\mathrm{Z}^{0}$}

The previous three Sections are replete with formalism, detailing the construction of an effective Lagrangian describing the Goldstone bosons associated with technicolour chiral symmetry breakdown. We conclude this paper by presenting some simple, yet phenomenologically interesting, applications of this formalism. We noted in Section 2 that some of the technicolour pseudo-Goldstone bosons (technions) should be light enough to be produced in decays of the $z^{0}$. We have seen already that the mass of the $Z^{0}$ is parametrically small compared to the 
scale $\mathrm{M}_{\mathrm{TC}}$; thus, $\mathrm{Z}^{0}$ decays lie in the regime to which our low energy phenomenology is applicable. We may compute amplitudes for the decays of $Z^{0}$ to technions using the effective Lagrangian").

The $Z^{0}$ width into a pair of these bosons is readily computed from the Feynman rules given in Fig. 1. Since technions have definite parity, they may be pair-produced only by the vector part of the current

$$
J_{3 L}^{\mu}-\sin ^{2} \theta_{w} \cdot J_{C \cdot m}^{\mu} .
$$

to which the $\mathrm{Z}^{0}$ couples. Thus, the partial width for pair production of any technion should be proportional to $\left(I_{3}-2 \sin ^{2} \theta_{W} Q\right)^{2}$. More precisely,

$$
\begin{aligned}
\Gamma\left(Z^{0} \rightarrow T \bar{T}\right)=\frac{\alpha m_{Z}}{12\left(\sin 2 \theta_{W}\right)^{2}} & \left(I_{3}-2 \sin ^{2} \theta_{W} Q\right)^{2} x . \\
& \times\left(1-\frac{4 m_{T}^{2}}{m_{Z}^{2}}\right)^{3 / 2} .
\end{aligned}
$$

For the production of the light technions $\mathrm{P}^{+} \mathrm{P}^{-}$, this width compares well with the width into two muons

$$
\frac{\Gamma\left(z^{0} \rightarrow p^{+} p^{-}\right)}{\Gamma\left(z^{0} \rightarrow \mu^{+} \mu^{-}\right)}=\frac{1}{2} \frac{\left(1-2 \sin ^{2} \theta_{W}\right)^{2}}{\left(1-4 \sin ^{2} \theta_{W}+8 \sin ^{4} \theta_{W}\right)}\left(1-\frac{4 m_{p}^{2}}{m_{z^{2}}^{2}}\right)_{(6.3)}^{3 / 2}
$$

If we ignore the phase space factor, the ratio $(6.3)$ is $(0.29)$ for $\sin ^{2} \theta_{W}=0.23$; $\mathrm{P}^{ \pm}$pairs should be copiously produced at the $\mathrm{Z}^{0}$. On the other hand, $\mathrm{P}^{0}$ and $\mathrm{P}^{\circ}{ }^{0}$, which have $Q=I_{3}=0$, are not pair-produced in $Z^{0}$ decays. We should note that this set of predictions is inescapable in technicolour models. These predictions provide then a clean test of the whole class of models we are considering.

We remarked in Section 4 on the absence of any term in the chiral Lagrangian coupling a technion to two gauge bosons. This implies that several reactions proposed 21$)$ to search for a neutral Higgs $\phi^{0}$, in particular, $Z^{0} \rightarrow \phi^{0} \mu^{+} \mu^{-}$, $\mathrm{Z}^{0} \rightarrow \phi^{0} \gamma$ and $e^{+} e^{-} \rightarrow \mathrm{Z}^{0} \phi^{0}$ are, for all practical purposes, absent in technicolour models. Small couplings of this type are induced by the axial vector anomalies of the currents associated with neutral technions, but these couplings, computed in Ref. 13), lead to partial widths for $Z^{0} \rightarrow \mathrm{P}^{0} \mu^{+} \mu^{-}, \mathrm{Z}^{0} \rightarrow \mathrm{P}^{0} \gamma$ of order $10^{-8}$ times the width to $\mu$ pairs.

*) Some of the results of this Section have been announced earlier in Ref. 17 ). 
One may, on the other hand, expect to see processes in which the $Z^{0}$ decays to more than two technions. Let us examine in detail the decay to three of these mesons. The amplitude for this process is given by the graphs of Fig. 3, where the vertices are those of the effective Lagrangian. (As before, we work in Landau gauge.) We remind the reader that the three Goldstone boson vertex required in the last two graphs comes only from the counterterm (5.8); the symmetric chiral Lagrangian (3.15) has no such vertex.

Let us work out these contributions explicitly for the case of a decay to three $\mathrm{P}$ mesons. For this, we will need the explicit forms of the corresponding generators of G. Set $\tau_{\alpha}=\left(1 / 21_{2}, \tau_{a}\right)$; this $\tau_{\alpha}$ includes an isosinglet matrix and the isospin generators. Then, for the particular SU and 0 models defined in Section 2, the four mesons $\mathrm{P}^{\alpha}$ correspond to generators $\mathrm{x}_{\mathrm{y}}$ of the form $(4.14)$ with

$$
g_{\alpha}=\tau_{\alpha} \otimes b
$$

where

$$
b=\frac{\sqrt{3}}{2} B \text {, }
$$

and $B$ is the matrix (2.6). Since the light $P$ mesons appear more generally in technicolour models, we should note that their $x_{y}$ are always of the form of (4.14) with (6.4), where $b$ is a matrix satisfying

$$
\operatorname{tr} b=0, \quad \operatorname{tr} b^{2}=1
$$

The second of these conditions is the normalization condition (4.12); the first is the condition that the generators corresponding to the $\mathrm{P}^{\alpha}$ be orthogonal to those corresponding to the fictitious Goldstone bosons $\pi^{\mathrm{a}}$.

The first graph of Fig. 3 requires a vertex coupling a gauge boson directly to three technions. Such a vertex appears in the second term of (4.11); by parity, it involves only the broken generator of $G$ to which the $Z^{0}$ couples

$$
\begin{aligned}
\mathcal{L}_{A-3 \pi}= & \frac{2}{3 f_{\pi}} \partial_{\mu} \pi_{x}(t \cdot \pi)_{x Z(A)}^{2} A_{\mu}^{A} \\
= & \frac{2}{3 f_{\pi}} \partial_{\mu} \pi_{x} \pi_{y} \pi_{z} A_{\mu}^{A} \times \\
& \times t_{r}\left(\left[\left[X_{x}, x_{y}\right], x_{z}\right] x_{z(A)}\right) .
\end{aligned}
$$

For the coupling of $\mathrm{Z}^{0}$ to $\mathrm{P}^{\prime} \mathrm{s}$, all the generators in (6.7) are diagonal of the form (4.14). Using the explicit forms of these generators, (4.25) for $X_{Z(A)}$ and $(6.4)$ for the $x_{y}$, we may recast. (6.7) as 


$$
\begin{aligned}
\delta \mathcal{L}_{Z-3 P}= & \frac{2}{3 f_{\pi}} \partial_{\mu} P_{\alpha} P_{\beta} P_{\gamma} \frac{e}{\sin 2 \theta_{W}} Z^{\mu} \times \\
& \times 2 \operatorname{tr}\left(\left[\left[\tau_{\alpha}, \tau_{\beta}\right], \tau_{\gamma}\right] \tau_{3}\right) \times \operatorname{tr} b_{3} .
\end{aligned}
$$

The isosinglet state $P^{\prime 0}$ gives no contribution to $(6.8)$. For the isotriplets $\mathrm{P}^{\mathrm{a}},(6.8)$ becomes

$$
\begin{aligned}
& \delta \mathcal{L}_{Z-3 P}=-\frac{2}{3 f_{\pi}} \partial_{\mu} P_{a} P_{b} P_{c} \frac{e}{\sin 2 \theta_{w}} Z^{\mu} \epsilon_{a b \alpha} \epsilon_{\alpha c 3} \cdot \operatorname{tr} b_{3} \\
& =\frac{2}{3} \frac{e \operatorname{tr} b_{3}}{f_{\pi} \sin 2 \theta_{w}} Z^{\mu}\left(\partial_{\mu} P_{3} P_{a} P_{a}-\partial_{\mu} P_{a} P_{a} P_{3}\right) .(6.9)
\end{aligned}
$$

Integrating by parts in the second term of (6.9), and discarding the surface term, we find

$$
\delta \mathscr{L}_{Z-3 P}=\frac{e t_{2} b_{3}}{f_{\pi} \sin 2 \theta_{w}} Z^{\mu} \cdot \partial_{\mu} P_{3} P_{a} P_{a},
$$

where, in (6.10), $a=1,2$ only. This interaction leads to the vertex shown in Fig. 4. For our SU and 0 models,

$$
\operatorname{tr} b_{3}=-1 / \sqrt{3} \text {, }
$$

but this parameter is, in general, sensitive to the number of techniquark and technilepton doublets.

To compute the contributions of the remaining diagrams, we need to work out the cubic coupling of three P's. Since, even in the 0 model, the P's correspond to generators of an $S U(N) \times S U(N)$ subgroup of $G$, we can use $(5.20)$ to compute this coupling. The sum over gauge bosons $A$ is most easily done in the original basis $\left(A_{\mu}^{\alpha}, w_{\mu}^{a}, B_{\mu}\right)$; the coupling matrices $G_{A}$ may be read from (2.5). Since the $P$ generators commute with the generators of colour, the $A_{\mu}^{\alpha}$ give no contribution to $P$ vertices. The $W_{\mu}^{a}$ have couplings which are purely left-handed; hence, these bosons give no contribution at all to (5.20). The boson $B_{\mu}$ has a non-trivial right-handed coupling, but its left-handed coupling involves only the generator $\left(I_{2} \times B\right)$, which commutes with (6.4). Hence, $(5.20)$ has no vertices at all involving only $P^{\prime} s$. This implies that $(5.20)$ contains no $P$ mass term; the result that the $P^{\prime}$ 's get no mass in order $\alpha$ is then contained in our formalism. However, it also implies that $(5.20)$ contains no 3-P vertex. The last two graphs of Fig. 3 simply vanish for the production of three $\mathrm{P}$ mesons. 
The non-vanishing vertex (6.10) itself embodies some interesting restrictions. We noted that this vertex involves only the broken part of the $Z^{0}$ current; this requires the three produced P's to sum to the three component of an isotriplet. However, the vertex (6.10) is yet more constrained; it allows, of all possible combinations of three $\mathrm{P}^{\prime} \mathrm{S}$, only the final state $\mathrm{P}^{0} \mathrm{P}^{+} \mathrm{P}^{-}$. From Fig. 4, we may compute the partial width for a decay into this state

$$
\begin{aligned}
& \Gamma\left(z^{0} \rightarrow P^{0} p^{+} p^{-}\right)=\frac{1}{2 m_{z}}\left(\frac{2 e t_{k} b_{3}}{f_{\pi} \sin 2 \theta_{w}}\right)^{2} \times \\
& \quad \times \int \frac{d^{3} k d^{3} d d^{3} q}{(2 \pi)^{9} 2 E_{k} 2 E_{p} 2 E_{q}} \cdot(2 \pi)^{4} \delta(k+p+q-Q)(k \cdot n)^{2},
\end{aligned}
$$

where $Q, k, p, q$ are the momenta of the $Z^{0}, P^{0}, P^{+}$and $\mathrm{P}^{-}$respectively, and $n_{\mu}=(0, \vec{n})$ is the polarization vector of the $z^{0}$. To get an idea of the magnitude of (6.12), let us set the masses of all three P's to zero; then the integrals may be readily evaluated:

$$
\left.\Gamma\left(z^{0} \rightarrow P^{0} P^{+} P^{-}\right)\right|_{m_{P}=0}=\frac{\alpha m_{z}}{\left(\sin 2 \theta_{W}\right)^{2}} \frac{\left(t_{r} b_{3}\right)^{2}}{768 \pi^{2}} \cdot\left(\frac{m_{z}}{f_{\pi}}\right)^{2},(6.13)
$$

that is,

$$
\left.\frac{\Gamma\left(z^{0} \rightarrow \rho^{0} p^{+} \rho^{-}\right)}{\Gamma\left(z^{0} \rightarrow \mu^{+} \mu^{-}\right)}\right|_{m_{p}=0}=\frac{\left(t_{2} b_{3}\right)^{2}}{128 \pi^{2}\left(1-4 \sin ^{2} \theta_{W}+8 \sin ^{4} \theta_{W}\right)}\left(\frac{m_{z}}{S_{\pi}}\right)^{2} .
$$

To evaluate (6.14), we choose $\sin ^{2} \theta_{W}=0.23$. Both $\left(\operatorname{Trb}_{3}\right)$ and $\left(\mathrm{m}_{\mathrm{z}} / \mathrm{f}_{\pi}\right)$ depend on the details of the technicolour model. However, if we choose the $\mathrm{sU}$ and 0 models of Section 2, we can use (4.21) and (6.11) and find

$$
\left.\frac{\Gamma\left(z^{0} \rightarrow \rho^{0} p^{+} p^{-}\right)}{\Gamma\left(z^{0} \rightarrow \mu^{+} \mu^{-}\right)}\right|_{m_{p}=0}=2.7 \times 10^{-4}
$$

Any technicolour model would give a result of the same order of magnitude. The ratio $(6.15)$ is small, but perhaps not unreasonably so in view of event rates expected at $L E P$ and elsewhere on the $Z^{0}$ resonance. This rate will be smaller of 
course if the $\mathrm{P}$ mesons have finite masses. To estimate this effect, allow the $\mathrm{P}^{ \pm}$to have finite mass, but, for simplicity, keep the $\mathrm{P}^{0}$ massless. Now (6.12) may be reduced to an integral over the momentum fraction carried by the $\mathrm{P}^{0}$

$$
x=2 k / m_{z},
$$

up to its maximum value

$$
x_{m}=1-\frac{4 m_{p}^{2}}{m_{z}^{2}} \text {. }
$$

One then finds

$$
\begin{aligned}
& \Gamma\left(z^{0} \rightarrow p^{0} p^{+} p^{-}\right)=\left.\Gamma\left(z^{0} \rightarrow p^{0} p^{+} p^{-}\right)\right|_{m_{p}=0} x \\
& \times 4 \int_{0}^{x_{m}} d x x^{3}\left(\frac{x_{m}-x}{1-x}\right)^{1 / 2} .
\end{aligned}
$$

This quantity is plotted as a function of the $\mathrm{P}^{+}$mass in Fig. 5 .

Whereas the width for $\mathrm{Z}^{0} \rightarrow \mathrm{P}^{+} \mathrm{P}^{-}$depended only on the quantum numbers of the $\mathrm{P}$ mesons, the process $\mathrm{Z}^{0} \rightarrow \mathrm{P}^{0} \mathrm{P}^{+} \mathrm{P}^{-}$involves, in a non-trivial way, the technicolour interactions through which the P's couple. This reaction provides us, already at the energy of the $\mathrm{Z}^{0}$, a chance to view directly particles interacting through the new strong force of technicolour.

\section{ACKNOWLEDGEMENTS}

Most of the work reported here was done while both authors were at the Centre d'Etudes Nucléaires de Saclay and we are very grateful for the hospitality extended to us there. We are particularly indebted to Professors Edouard Brézin and Jean Zinn-Justin for imparting to us some of their insights. We are also grateful to Predrag Cvitanović and Paul Ginsparg for useful suggestions, and to John Ellis who repeatedly read the manuscript and made incisive comments. One of us (M.E.P.) gratefully acknowledges financial support from the Harvard Society of Junior Fellows. 


\section{APPENDIX - GROUP THEORETICAL CONVENTIONS}

We imagine a group $G$ broken to a subgroup $H$. We label the generators of $G$ by $G_{a}$, the generators of $H$ by $T_{i}$ and the remaining, broken generators of $G$ by $x_{y}$. We use letters from the beginning of the alphabet $(a, b, c)$ to index the $G$, letters from the middle of the alphabet $(i, j)$ to index the $T$ and letters from the end of the alphabet $(x, y)$ to label the $x$. Generators of $\mathrm{G}$ are normalized to

$$
\operatorname{tr}\left(G_{a} G_{b}\right)=\delta_{a b} \text {. }
$$

The structure constants of $G$ are defined by

$$
\left[G_{a}, G_{b}\right]=t_{a b c} G_{c} \text {. }
$$

For $G=S U(N) \times S U(N)$ or $G=S U(2 N)$, it is useful to consider the diagonal SU(N) subgroup. We label the generators of this subgroup by $g_{\alpha}$ and assign them Greek indices $(\alpha, \beta, \gamma)$. These are normalized to

$$
t_{2}\left(g_{\alpha} g_{\beta}\right)=\frac{1}{2} \delta_{\alpha \beta}
$$

Generators of $G$ are constructed from the $g_{\alpha}$ according to (4.14). In discussing the couplings of gauge mesons, it is useful to work with generators of $G$ which are normalized, not according to (A.I), but in some other way which simplifies the form of this coupling. Such generators are indexed by capital letters: $G_{A}$. The decomposition of such a generator into unbroken and broken pieces is denoted by writing

$$
G_{A}=I_{I(A)}+X_{Z(A)}
$$

or simply by the use of $I(A)$ or $Z(A)$ as an index. The structure constant with a capitalized index is defined by

$$
t_{A b C}=\operatorname{tr}\left(G_{A}\left[G_{b}, G_{c}\right]\right)
$$

We denote the $2 \times 2$ generators of isospin and the $3 \times 3$ generators of colour by $\tau_{a}$ and $\lambda_{\alpha}$ respectively and normalize them to

$$
t_{2}\left(\tau_{a} \tau_{b}\right)=\frac{1}{2} \delta_{a b}, \quad t_{r}\left(\lambda_{\alpha} \lambda_{\beta}\right)=\frac{1}{2} \delta_{\alpha \beta} .
$$

The $n \times n$ unit matrix is denoted $I_{n} \cdot 8 \times 8$ SU(8) matrices are built from these by taking direct products: for example, $\tau_{a} \times 1_{4}$ is an $8 \times 8$ matrix acting on isospin. 
REFERENCES

1. S. Weinberg, Phys. Rev. Lett. 19 (1967) 1264;

A. Salam, in Elementary Particle Theory, ed. N. Svartholm (Almquist and Wiksell, Stockholm, 1968).

2. S. Weinberg, Phys. Rev. D13 (1967) 974.

3. S. Weinberg, Phys. Rev. D19 (1979) 1277;

L. Susskind, Phys. Rev. D20 (1979) 2619.

4. E. Eichten and K.D. Lane, Phys. Lett. 90B (1980) 125.

5. S. Dimopoulos and L. Susskind, Nucl. Phys. B155 (1979) 237.

6. S. Weinberg, Phys. Rev. Lett. 18 (1967) 188;

J. Schwinger, Phys. Lett. 24B (1967) 473.

7. S. Coleman, J. Wess and B. Zumino, Phys. Rev. 177 (1969) 2239;

C.G. Callan, S. Coleman, J. Wess and B. Zumino, Phys. Rev. 177 (1969) 2247.

8. J. Schwinger, in Proceedings of the Seventh Hawaii Topical Conference in Particle Physics, ed. R.J. Cence, P.N. Dobson, S. Pakvasa and S.F. Tuan (University Press of Hawaii, 1978).

9. S. Weinberg, Physica 96A (1979) 327.

10. M.A.B. Bég, H.D. Politzer and P. Ramond, Phys. Rev. Lett. 43 (1979) 1701.

11. S. Dimopoulos, Nucl. Phys. B168 (1980) 69.

12. S. Dimopoulos, S. Raby and G.L. Kane, University of Michigan preprint UM HE $80-$ 22 (1980).

13. J. Ellis, M.K. Gaillard, D.V. Nanopoulos and P. Sikivie, LAPP preprint TH 23 and CERN preprint TH 2938 (1980);

A. Ali and M.A.B. Bég, DESY preprint 80/98 (1980).

14. S. Chadha and M.E. Peskin, CERN preprint in preparation.

15. M.E. Peskin, Nucl. Phys. BI75 (1980) 197.

16. J.P. Preskill, Nucl. Phys. B177 (1981) 21.

17. K.D. Lane and M.E. Peskin, in Proceedings of the XV Rencontre de Moriond, Vol II, Electroweak Interactions and Unified Theories, ed. J. Tran Thanh Van (Editions Frontières, France 1980).

18. P. Sikivie, Lectures given at the 1980 Varenna Summer School, CERN preprint TH 2951 (1980).

19. P. Sikivie, L. Susskind, M. Voloshin and V. Zakharov, Nucl. Phys. B173 (1980)

20. S. Dimopoulos, S. Raby and P. Sikivie, Stanford University preprint ITP 664 (1980).

21. J.D. Bjorken, in Proceedings of the 1976 SLAC Summer Institute on Particle Physics SLAC 198 (1977);

R.N. Cahn, M.S. Chanowitz and N. Fleishon, Phys. Lett. $82 B$ (1979) 113;

G. Barbiellini et al., DESY preprint $79 / 27$ (1979). 


\section{FIGURE CAPTIONS}

Fig. I : Some couplings of Goldstone bosons to gauge bosons which arise from the chiral Lagrangian.

Fig. 2 : Diagrams with virtual $\mathrm{G}_{\mathrm{W}}$ gauge bosons producing $\mathrm{G}$ violating corrections to the Goldstone boson effective Lagrangian.

Fig. 3 : Contributions to the amplitude for the decay of the $\mathrm{Z}^{0}$ into three technions.

Fig. 4 : Feynman rule for the coupling of the $Z^{0}$ to three $P$ mesons.

Fig. $5: \Gamma\left(2^{0} \rightarrow \mathrm{P}^{0} \mathrm{P}^{+} \mathrm{P}^{-}\right)$in the $\mathrm{SU}$ and 0 models of Section 2 , as a function of the mass of the $\mathrm{P}^{ \pm}$, expressed as a fraction of $\Gamma\left(Z^{0} \rightarrow \mu^{+} \mu^{-}\right)$. The $\mathrm{P}^{0}$ mass has been neglected. We have used $m_{\mathrm{Z}}=88 \mathrm{GeV}$. 

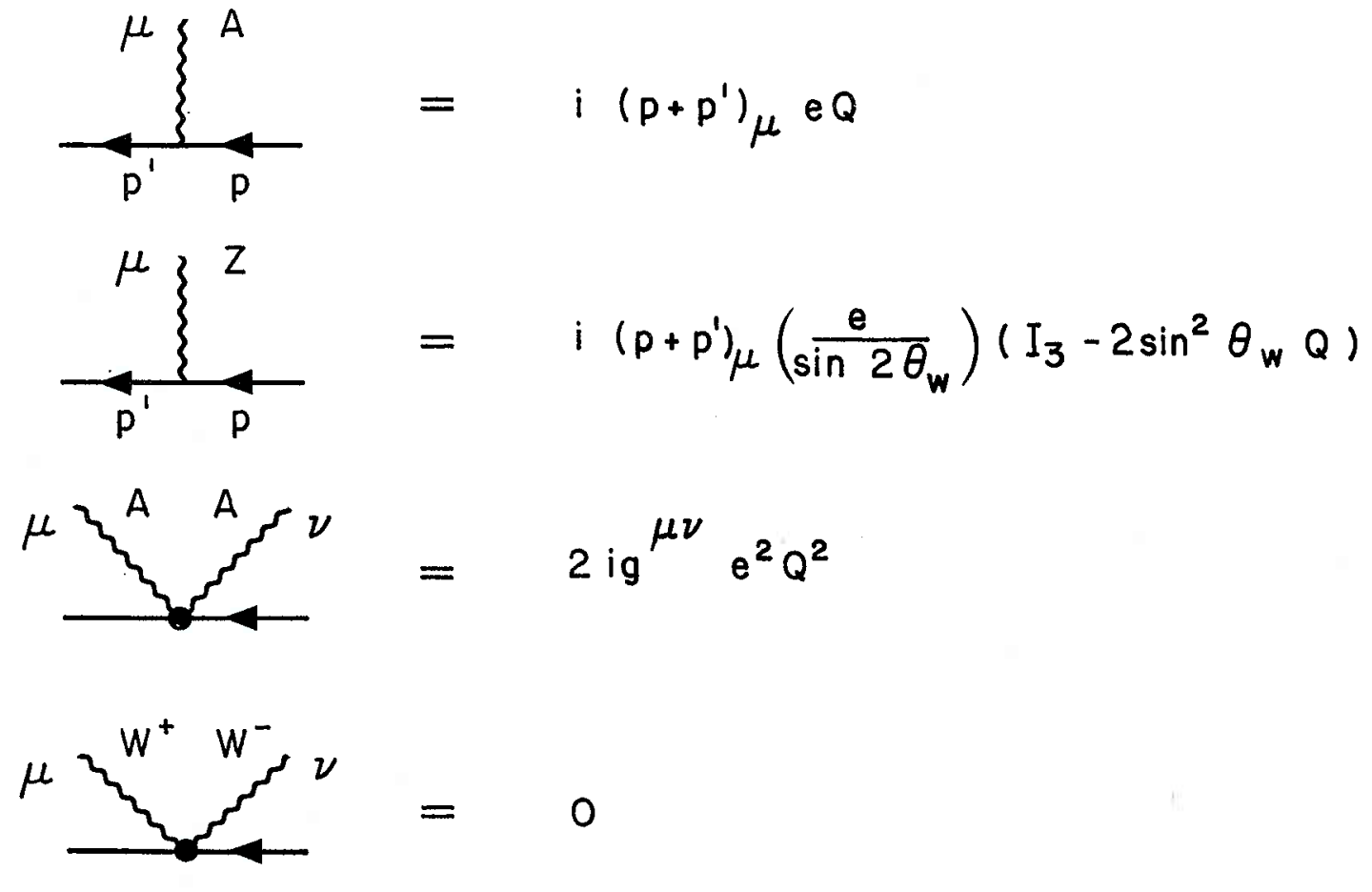

$$
\mu \underbrace{Z_{2}^{Z} Z^{Z} \nu}=-2 \mathrm{ig}^{\mu \nu} \frac{\mathrm{e}^{2} \mathrm{Q}}{\cos ^{2} \theta_{w}}\left(I_{3}-\sin ^{2} \theta_{w} Q\right)
$$

Fig.1 

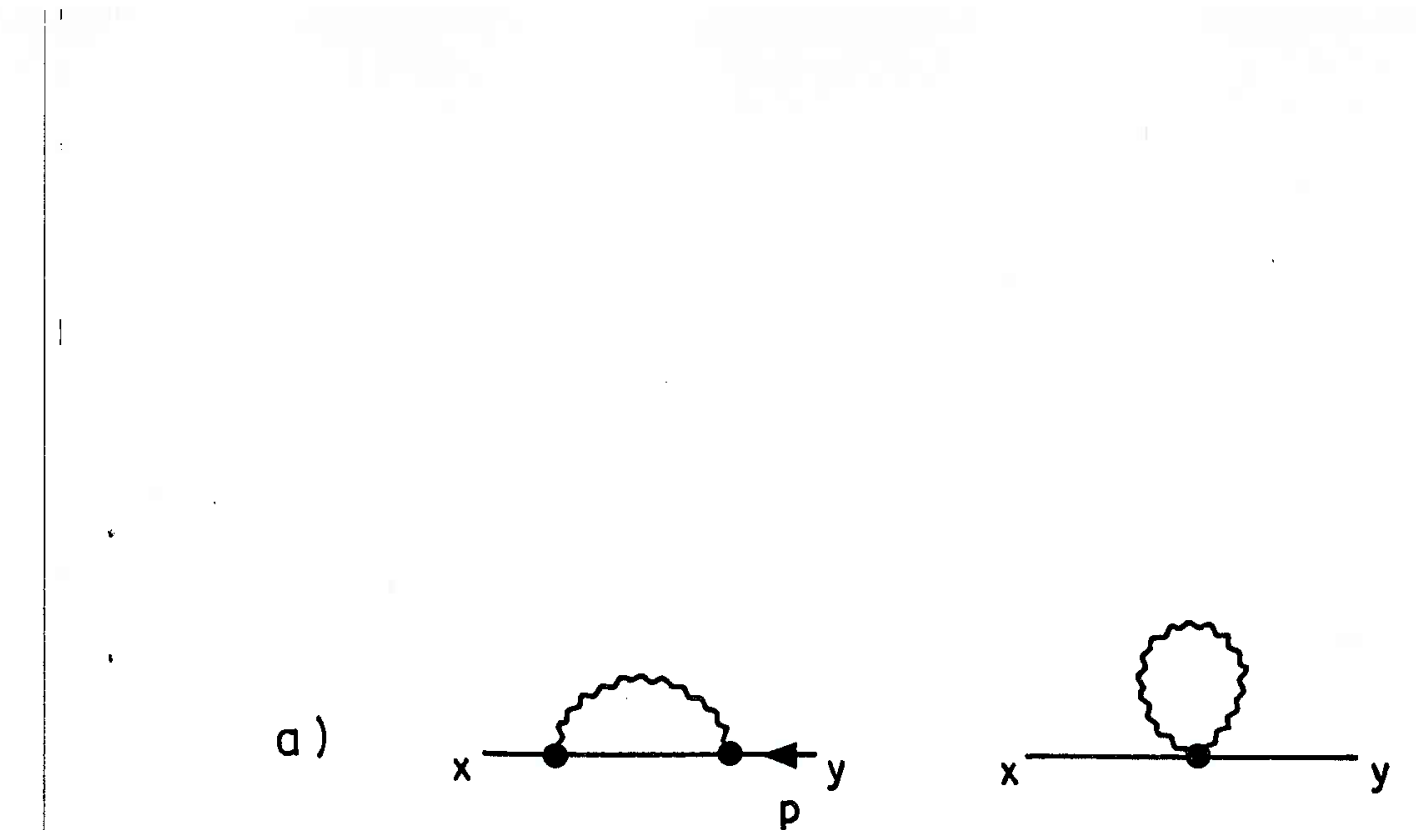

b)
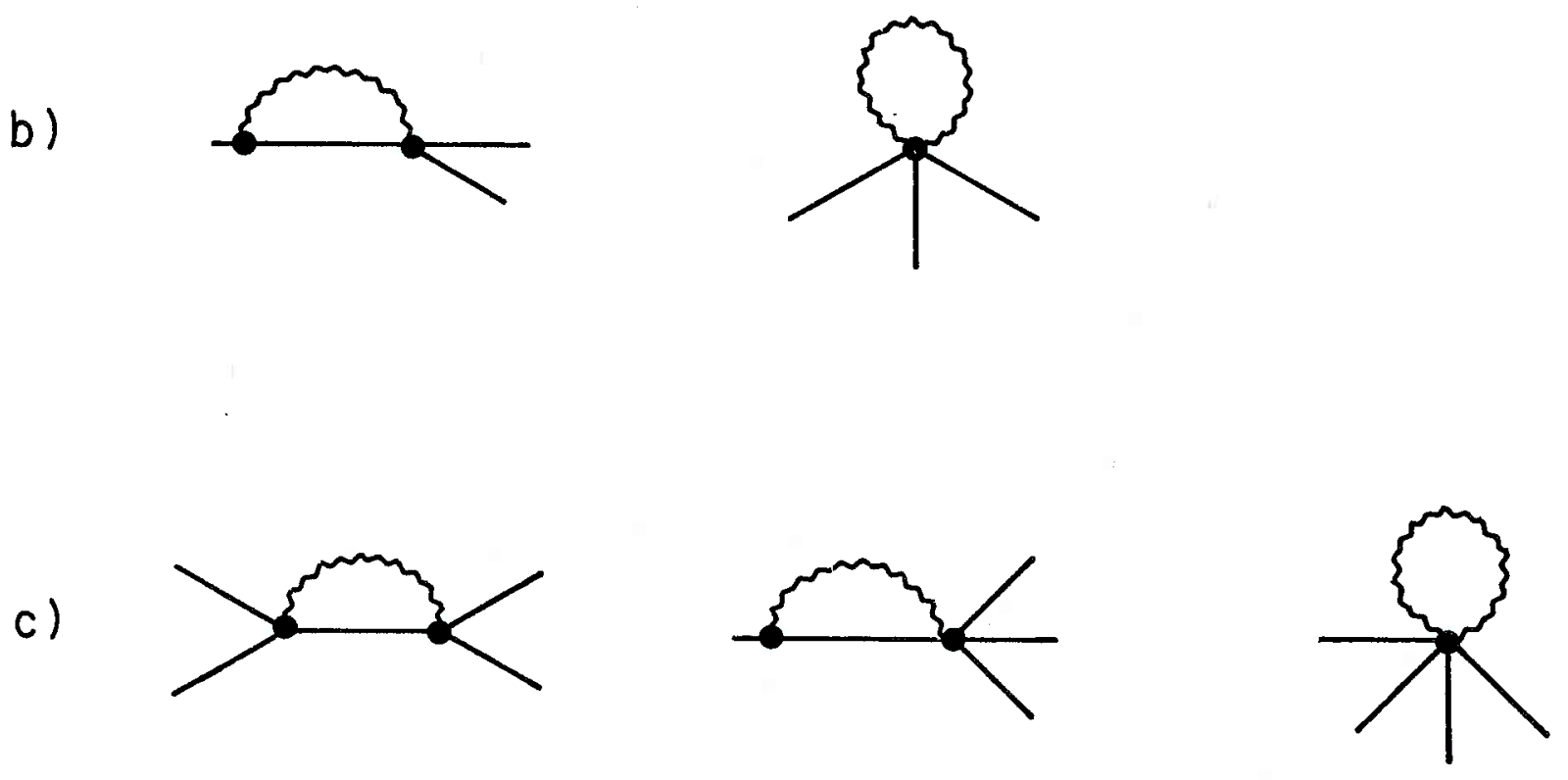

Fig. 2 

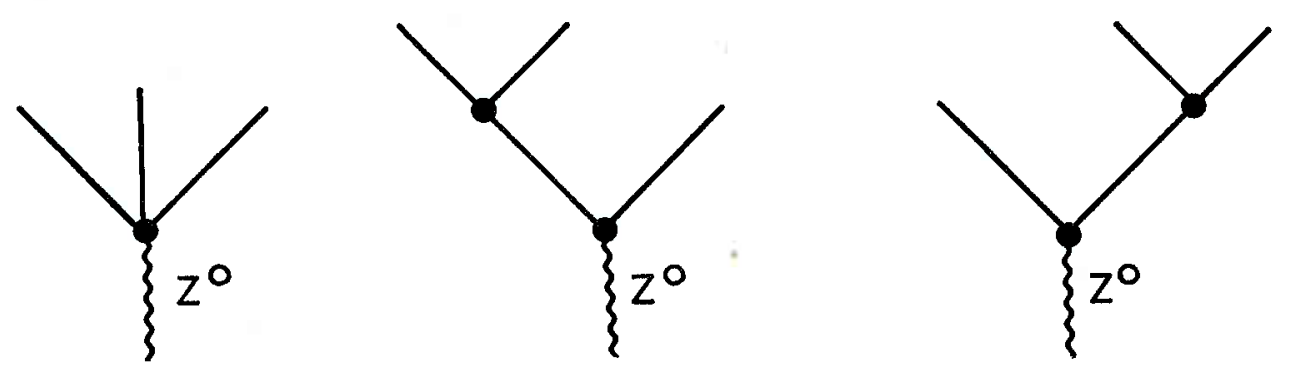

Fig. 3

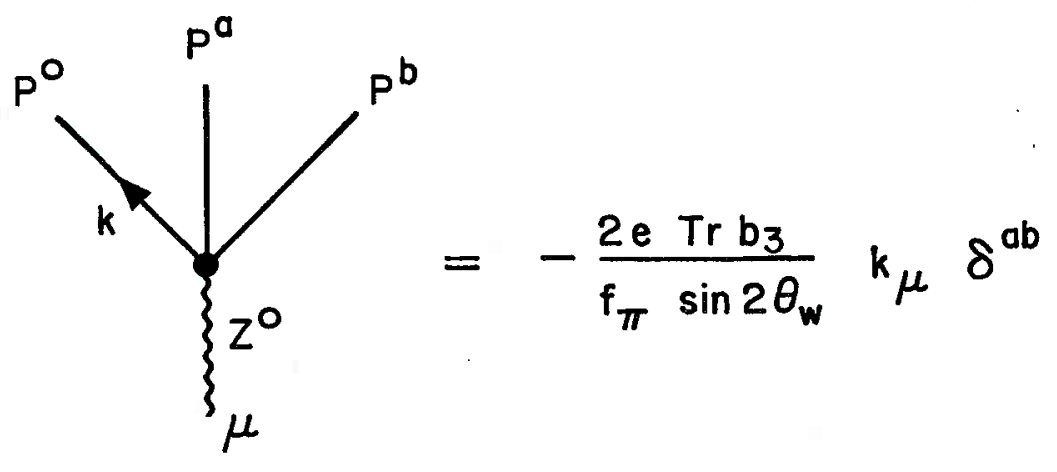

Fig. 4 


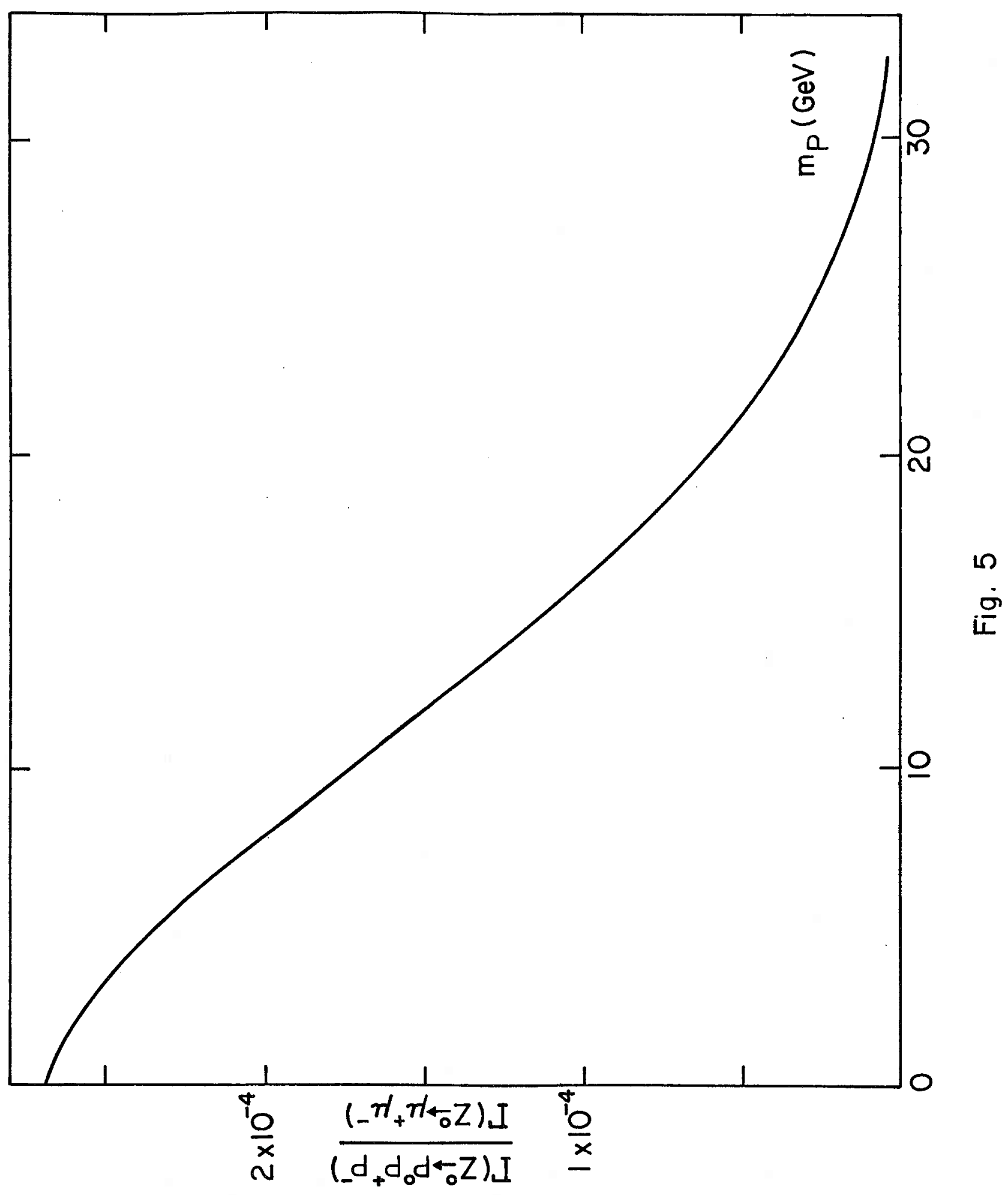

